\title{
Spectroscopic signatures of the vanishing natural coronagraph of Eta Carinae
}

A. Damineli ${ }^{\circledR},{ }^{1 \star}$ F. Navarete ${ }^{\circledR},{ }^{1 \star}$ D. J. Hillier ${ }^{\circledR}, 2$ A. F. J. Moffat ${ }^{\circledR},{ }^{3}$ M. F. Corcoran ${ }^{\circledR},{ }^{4,5}$ T. R. Gull ${ }^{\circledR}, 6$ N. D. Richardson ${ }^{\circledR},{ }^{7}$ G. Weigelt ${ }^{\circledR}, 8$ P. W. Morris ${ }^{\oplus 9}$ and I. Stevens ${ }^{\circledR 10}$

${ }^{1}$ Universidade de São Paulo, Instituto de Astronomia, Geofísica e Ciências Atmosféricas, Rua do Matão 1226, Cidade Universitária São Paulo-SP, 05508-090, Brasil

${ }^{2}$ Department of Physics and Astronomy \& Pittsburgh Particle Physics, Astrophysics, and Cosmology Center (PITT PACC), University of Pittsburgh, 3941 O'Hara Street, Pittsburgh, PA 15260, USA

${ }^{3}$ Département de Physique and Centre de Recherche en Astrophysique du Québec (CRAQ) Université de Montréal, C.P. 6128, Succ. Centre-Ville, Montréal, Québec H3C 3J7, Canada

${ }^{4}$ CRESST II \& X-ray Astrophysics Laboratory, Code 662, NASA Goddard Space Flight Center, Greenbelt, MD 20771, USA

${ }^{5}$ Department of Physics The Catholic University of America, Institute for Astrophysics and Computational Sciences, Washington, DC 20064, USA

${ }^{6}$ Laboratory for Extraterrestrial Planets and Stellar Astrophysics, Code 667, NASA Goddard Space Flight Center, Greenbelt, MD 20771, USA

${ }^{7}$ Department of Physics and Astronomy, Embry-Riddle Aeronautical University, 3700 Willow Creek Road, Prescott, AZ 86301, USA

${ }^{8}$ Max Planck Institute for Radio Astronomy, Auf dem Hügel 69, Bonn D-53121, Germany

${ }^{9}$ California Institute of Technology, IPAC M/C 100-22, Pasadena, CA 91125, USA

${ }^{10}$ School of Physics \& Astronomy, University of Birmingham, Edgbaston, Birmingham B15 2TT, UK

Accepted 2021 May 10. Received 2021 May 10; in original form 2021 February 28

\section{ABSTRACT}

Eta Carinae is a massive interacting binary system shrouded in a complex circumstellar environment whose evolution is the source of the long-term brightening observed during the last $80 \mathrm{yr}$. An occulter, acting as a natural coronagraph, impacts observations from our perspective, but not from most other directions. Other sight-lines are visible to us through studies of the Homunculus reflection nebula. The coronagraph appears to be vanishing, decreasing the extinction towards the central star, and causing the star's secular brightening. In contrast, the Homunculus remains at an almost constant brightness. The coronagraph primarily suppresses the stellar continuum, to a lesser extent the wind lines, and not the circumstellar emission lines. This explains why the absolute values of equivalent widths (EWs) of the emission lines in our direct view are larger than those seen in reflected by the Homunculus, why the direct view absolute EWs are decreasing with time, and why lower-excitation spectral wind lines formed at larger radii (e.g Fe II $4585 \AA$ ) decrease in intensity at a faster pace than higher excitation lines that form closer to the star (e.g. $\mathrm{H} \delta$ ). Our main result is that the star, despite its 10-fold brightening over two decades, is relatively stable. A vanishing coronagraph that can explain both the large flux evolution and the much weaker spectral evolution. This is contrary to suggestions that the long-term variability is intrinsic to the primary star that is still recovering from the Great Eruption with a decreasing mass-loss rate and a polar wind that is evolving at a slower pace than at the equator.

Key words: stars: binaries - stars: individual: eta Carinae-stars: winds-ISM: dust, extinction-ISM: molecules .

\section{INTRODUCTION}

The evolution and fate of massive stars are often determined by binary interaction (Sana et al. 2012). Their scarcity in the solar neighbourhood makes progress in the field difficult. $\eta$ Car is relevant because of its proximity $(2.3 \mathrm{kpc})$, its long observational history, and because it is one of the most massive stars within our Galaxy. It owes its fame to the Great Eruption (GE) that occurred in the 1840s, when it became the second brightest star in the night sky and ejected the elegant bipolar Homunculus nebula (Davidson \& Humphreys 1997). Hidden behind circumstellar dust (Andriesse, Donn \& Viotti 1978; Viotti et al. 1989), the central object is a highly eccentric massive binary (Damineli 1996; Damineli, Conti \& Lopes 1997; Damineli

^ E-mail: augusto.damineli@gmail.com (AD); navarete@usp.br (FN) et al. 2000) with periastron passages occurring every $5.538 \mathrm{yr}$ and that are monitored throughout the electromagnetic spectrum (Pittard \& Corcoran 2002; Duncan \& White 2003; Damineli et al. 2008; Fernández-Lajús et al. 2010; Mehner et al. 2014; Teodoro et al. 2016; Corcoran et al. 2017).

The primary star ( $\eta$ Car A) dominates the optical light of the system. Its extinction-corrected apparent magnitude would be $V=$ $0.94 \mathrm{mag}$, according to modelling of the optical/UV spectrum (Hillier et al. 2006), plus a foreground extinction of up to $A_{\mathrm{V}}=2.5 \mathrm{mag}$ (Teodoro et al. 2020). The apparent magnitude of the central stellar core was $V=7.73$ on 1999.140 (Martin \& Koppelman 2004), indicating additional local extinction obscuring our view. If the dust was uniformly distributed around the stellar core, the Homunculus reflection nebula would be proportionately fainter. The simple fact that the Homunculus is so prominent, compared to the central star, indicates extra extinction in our line of sight (LOS) to the central 
star. Moreover, the relative high brightness of the three speckle objects BCD (Weigelt \& Ebersberger 1986) could be explained if they are illuminated by the central star and seen in an unimpeded view away from our LOS, while the central star, located a fraction of an arcsecond away, is seen under heavy extinction caused, for example, by an obscuring dusty disc (Weigelt et al. 1995) or some other optically thick, localized structure.

From the 1940s through the 1990s, the $\eta$ Car system (star + Homunculus) had continuously brightened at a rate of $\delta V \sim-0.02 \mathrm{mag} \mathrm{yr}^{-1}$ in the $V$ band. However, existing imagery from those epochs cannot separate the stellar contribution from the nebula. Over the past two decades, the brightening increased to a rate of $\delta V \sim-0.05 \mathrm{mag} \mathrm{yr}^{-1}$ (Martin, Davidson \& Koppelman 2006), and improved photometric imagery allowed the separation of the stellar core from the flux of the Homunculus nebula: the stellar core brightened at a rate $\delta V \sim-0.11 \mathrm{mag} \mathrm{yr}^{-1}$ while the Homunculus nebula has remained fairly constant at $V=5.48 \pm 0.05 \mathrm{mag}$ (Damineli et al. 2019). The light emitted by the central star and reflected in the NW extremity of the Homunculus reaches the Earth with a delay of $\sim 6$ months. If the star was intrinsically brightening, the Homunculus light curve should show the same effect delayed by a few months. In 2010 the brightness of the central star surpassed that of the Homunculus in the $V$ band for the first time in $160 \mathrm{yr}$. Since the Homunculus is a reflection nebula and is almost constant in brightness, the extinction along our LOS to the central star must be changing (Damineli et al. 2019). The brightening cannot be explained by the central star becoming intrinsically more luminous since the stellar spectrum has not evolved significantly even though the object brightened by approximately a factor of 10 in the last $70 \mathrm{yr}$. No such long-term brightening was observed in the mid-infrared, as reported by Mehner et al. (2019). The sparse observations in the mid-infrared since 1968 are compatible with lower amplitude variations (factor 2) on a time-scale of years, as is the case also for the orbital modulation in the optical reported by Damineli et al. (2019). The mid-infrared flux is produced by the absorption and re-emission of light from the central object by dust spread over the whole nebula. In contrast to the optical and ultraviolet emission, it is extended, and not affected by any localized structure.

$\eta$ Car is a strong X-ray source in which thermal X-rays are produced in the hot shocked gas where the wind from the primary star collides with the wind of the companion. The luminosity and temperature of the X-ray emission depend on the wind properties [i.e. massloss rates and pre-shock wind velocities; see for example Stevens, Blondin \& Pollock (1992)]. The wind parameters in turn depend on the underlying stellar parameters. Thus, variations in the intrinsic stellar properties would be reflected in variations in the observed Xray spectrum. The X-ray temperature and luminosity near apastron (when Coriolis distortions are minimal) show little variations from orbit-to-orbit over the last four orbits for which detailed monitoring data exist - see Corcoran et al. (2017) and Espinoza Galeas et al. (2021) - indicating that neither the wind nor stellar parameters have varied significantly during the prior two decades. This conclusion is supported by the lack of variations in the terminal velocities derived from the P-Cygni absorption profiles of the upper members of the Balmer series (like $\mathrm{H} \delta$ ) (Teodoro et al. 2012).

The initial suggestion that we see the central star obscured by dust condensation and that the Homunculus scatters radiation that has suffered relatively little attenuation was made by Hillier \& Allen (1992). This idea arose because the equivalent widths (EWs) of the lines seen in direct light from the central star were larger than the corresponding EWs measured in reflection from the Homunculus. This was true for some wind lines (Fe II, HI), and particularly true for the narrow nebular lines. Indeed, the reflected spectrum was much more similar to a classic P Cygni spectrum than the spectrum of $\eta$ Car observed in direct light. Hillier et al. (2006) discussed additional evidence of the presence of a kind of natural coronagraph in $\eta$ Car.

Based on spatially resolved HST/STIS spectroscopy of the star, Hillier et al. (2006) showed that the spectrum of $\eta$ Car could be modelled only if the star and its wind suffered a visual extinction $(\approx 7$ mags at $\mathrm{V})$ that was much larger than could be attributed to the interstellar medium ( $<2$ mags). However, the models of Hillier et al. (2006) always underestimate the observed EWs of some emission lines such as $\mathrm{H} \alpha$ and $\mathrm{H} \beta$. The inability to match these lines can be attributed to the presence of an occulter that reduces the continuum flux more than the line flux.

The presence of the coronagraph explains the unusual spectra taken in the 1980s and 1990s using ground-based spectrographs. The spectra exhibited broad hydrogen and permitted iron lines characteristic of a typical P Cygni star with a terminal velocity of $\sim 500 \mathrm{~km} \mathrm{~s}^{-1}$ (Zanella, Wolf \& Stahl 1984; Hillier \& Allen 1992). However, the spectrum also showed narrow forbidden lines of $\mathrm{Fe}$ superimposed on broad profiles. The narrow forbidden lines arise in partially ionized structures including the three Weigelt knots (Weigelt \& Ebersberger 1986; Davidson et al. 1995), while the broad components arise in the very extended wind (Hillier et al. 2001). Normally the wind components would be too faint to be seen. However, the coronagraph systematically weakens the stellar continuum, but less so the broad components from the very extended wind. As the coronagraph vanishes, the stellar continuum emission increases and hence the extended broadened wind lines and the narrow forbidden lines, which have constant flux, become less prominent (Davidson et al. 2005; Mehner et al. 2010b).

Mehner et al. (2012) made detailed monitoring of a set of spectral lines in the period 1998-2011, in direct light to the star and also at the FOS4 position (Davidson et al. 1995) near the SE pole of the Homunculus. In addition to confirming the weakening of the wind lines of the central star, that work showed that the broad-line absolute EWs formed in the primary's wind decreased at a faster pace than those observed in reflected light at FOS4. Part of the present work is focused on the EW evolution of the three representative lines of the stellar wind ( $\mathrm{H} \alpha, \mathrm{H} \delta$, and Fe II $4585 \AA$ ) studied by Mehner et al. (2012), extending the data set to the time interval 1990-2021 (see Fig. 4). The observational results of Mehner et al. (2012), made during a shorter time interval, were confirmed, although the physical mechanisms suggested to explain the observations differ from those presented here.

Mehner et al. (2012) suggested that the secular evolution of the spectra is due to variations intrinsic to the primary star that is still recovering from the $1840 \mathrm{~s}$ GE. They suggest that the mass-loss rate is decreasing, making the wind more transparent. However, since the evolution of the spectral lines from the star and the corresponding ones at FOS4 are different, Mehner et al. (2012) postulate that the stellar wind at the equator is evolving differently from that at the pole. While the P Cygni absorption component can be strongly dependent on viewing angle, the emission component in a moderately aspherical wind will vary much less, simply because one is averaging the emission over a large volume. Thus, it is unlikely that a simple latitudinal variation in mass-loss rate could explain the observed variations. Further, this model, by itself, does not explain the large changes in the apparent brightness of $\eta$ Car.

Recent ALMA observations have also provided direct confirmation of the coronograph at sub-arcsecond resolutions $(<0$ '.5 $\sim 1100 \mathrm{au}$ ). Although ALMA mapped molecular absorption lines, they should trace the warm/cold dust. Bordiu \& Rizzo (2019) 
found $\mathrm{CO}\left(-9 \mathrm{~km} \mathrm{~s}^{-1}\right), \mathrm{HCN}$ (Hydrogen cyanide), and $\mathrm{H}^{13} \mathrm{CN}$ $\left(-60 \mathrm{~km} \mathrm{~s}^{-1}\right)$ in absorption in front of the star at $T \sim 500 \mathrm{~K}$. Morris et al. (2020) found an absorption spot they attributed to $\mathrm{CH}_{3} \mathrm{OH}$ (Bordiu \& Rizzo 2019) and another of ${ }^{12} \mathrm{CO}$ at radial velocity $+3 \pm 5 \mathrm{~km} \mathrm{~s}^{-1}$ in front of the star at $T \sim 100-110 \mathrm{~K}$. Different velocities and temperatures suggest that the absorbing material (inside the occulter) are spread at different radii in front of the star. These molecular absorption lines should be fading, but only future visits by ALMA can test this hypothesis.

In this work, we present new data and analyse spectroscopic evidence for the existence of a natural coronagraph in front of $\eta$ Car. The paper is organized as follows: Section 2 presents the data; Section 3 presents our results; in Section 4 we discuss these results; Section 5 presents our conclusions.

Through this paper we use the binary ephemerides reported by Teodoro et al. (2016): period $=2022.7 \mathrm{~d}$, phase zero $=$ HJD 2456874.4. Following Grant, Blundell \& Matthews (2020) the actual periastron passage occurs $4 \mathrm{~d}$ earlier than this definition of phase zero. The numbering of the orbital cycles was defined by Groh \& Damineli (2004).

\section{OBSERVATIONS}

We have collected a large number of medium/high-resolution spectra over the past $30 \mathrm{yr}$ from different observatories, by different observers, and reported in a number of papers.

The reader should bear in mind that we do not aim to present a general description of the spectral features in $\eta \mathrm{Car}-$ this has been done in earlier works. We selected the data by focusing on a specific question: Is there any strong support for the existence of a natural coronagraph in $\eta$ Car? Consequently, we do not explore spectral features and particular modelling results that are interesting but a distraction from our goal.

Homogeneity of the data is a major concern in a large data set. Also of major concern when measuring EWs and line fluxes is the definition of the stellar continuum. This is particularly difficult in $\eta$ Car since the lines are relatively broad, frequently display extended wings, and are often blended. Échelle observations can exacerbate the problem further because of the difficulty in joining orders. Signal-to-noise ratio $(\mathrm{S} / \mathrm{N})$ and spectral resolving power $(\mathrm{R})$ are important for measuring line profiles, less so for measuring EWs. For measuring EWs, the wavelength integration limits are important, although subject to a somewhat arbitrary choice, due to blends with neighbour lines. This can be controlled by always using the same limits. The blended lines are much fainter and insert a low-level bias in the time series. The seeing also is not too critical for the central star in $\eta$ Car, since most of the emitting structures are confined to the central 0.5 arcsec, and the nebular contribution is far smaller.

As a quality check on measurements, we have compared slit spectra taken at 1-1.5 arcsec seeing with FEROS fiber-fed (diameter 2.7 $\operatorname{arcsec}$ ) and found no important line profile differences. We made extractions of the stellar spectrum in a typical UVES spectrum [seeing full width at half-maximum $(\mathrm{FWHM}) \sim 0.8 \mathrm{arcsec}]$ using apertures starting from $0.5 \operatorname{arcsec}(2$ pixels) up to $2 \operatorname{arcsec}(8$ pixels) and found no significant differences in the normalized spectra. We then defined 1.0 arcsec as the length along the slit to extract the UVES spectra. In order to check our procedure, we compared our measurements with those published in Mehner et al. (2012) and found reasonable agreement. Spectra from the star taken with different ground-based spectrographs coeval with UVES observations also showed good agreement without applying any adjustment shifts in EWs.
Good image quality is of major importance for the nebular emission, as is the scattered light internally to the spectrograph. For this task, the UVES data set taken at the FOS4 position is crucial.

HST/STIS spectra resolve the structures around the central star, which demands special care in extracting the spectra to be compared with ground-based observations. For the sake of homogeneity, since they do not exhibit a dense time sampling or long-term monitoring as the ground-based data, we did not use the HST data.

For the purpose of the present work, the main sources of data are described below.

(i) UVES direct and reflected light spectra: We selected high $\mathrm{R}$ and S/N spectra observed at VLT/UVES/ESO-Chile with $R \sim 100000$. The data were retrieved from the $\eta$ CarTreasury Program ${ }^{1}$ archive and were used to study the difference between direct and reflected-light spectra. We extracted UVES 1D spectra by tracing the $2 \mathrm{D}$ spectra within \pm 2 pixels $(1 \mathrm{arcsec})$ from the slit center. The slit width was 0.4 arcsec. We used coeval stellar and FOS4 spectra (when available) with the same parameters to extract the nebular spectra. Most of the UVES spectra at FOS4 (Davidson et al. 1995; Mehner et al. 2012) in the period 1999.97-2009.50 were centered at $\sim 2.84 \operatorname{arcsec} \mathrm{E}$ and $\sim-2.36 \operatorname{arcsec} \mathrm{S}$ of the central star $(3.7$ arcsec SE). Unfortunately, after 2013 this position was missed by the telescope pointing and the slit center was not in coincidence with FOS4. As shown in Mehner et al. (2012), line profiles change from point to point. Although extracted the few nebular spectra in 2013-2015 at $>0.5$ arcsec off the slit center, we did not use these in the formal analysis. EWs from FOS4 spectra are listed in Table A6.

A pair of spectra in direct and reflected light was taken in 2006 May/June, separated by $18 \mathrm{~d}$, both during the high-excitation phase when the cyclical variability arising from the $5.538 \mathrm{yr}$ period was at a minimum - to extract the representative line profiles (they are shown in Fig. 1). The observations of the direct spectrum were obtained on $\mathrm{HJD}=2453894.5$, and for the reflected spectrum at the FOS4 position on HJD $=2453866.5$. Although not simultaneous with the reflected spectra, the inspection of $\mathrm{H} \alpha$ spectra taken in direct light at several observatories in the time frame 2005.5-2006.5 confirmed that $\mathrm{H} \alpha$-EW was stable at better than 5 per cent.

(ii) Optical spectra taken with the FEROS fiber-fed spectrograph $(R \sim 48000)$ at the 0.52 and 2.2-m telescopes at La Silla Observatory (ESO-Ch): The spectra were obtained in the time-frame 1992-1999 (and references therein Damineli et al. 2000) and a few others in subsequent years. In these spectra, we measured EWs for $\mathrm{H} \alpha, \mathrm{H} \delta$, and Fe II $4585 \AA$. The fiber diameter projected on to the sky was 2.7 arcsec.

(iii) Optical spectra taken with the CTIO 1.5-m telescope with two different spectrographs: CHIRON/CTIO $(R \sim 90000)$ in the period 2009-2021 and the previous version of this spectrograph, a fiber-fed echelle with $R \sim 40000$; as described by Richardson et al. (2010, 2015, 2016), Mehner et al. (2012), and Teodoro et al. (2016).

(iv) The SOAR/Goodman spectrograph was used in several runs from 2008 to 2014 in the blue part of the spectrum, with $R \sim 3500$ (Teodoro et al. 2016).

(v) Optical spectra collected with NRES/LCOGT at South Africa and CTIO with $R \sim 48000$. The fiber diameter projected on to the sky was 2.8 arcsec (Navarete et al., in preparation). The 2019.9 LCO spectrum was used to compare with the 1999.1 FEROS spectrum listed in columns 5 and 6 of Table 2 .

\footnotetext{
${ }^{1}$ http://etacar.umn.edu/
} 


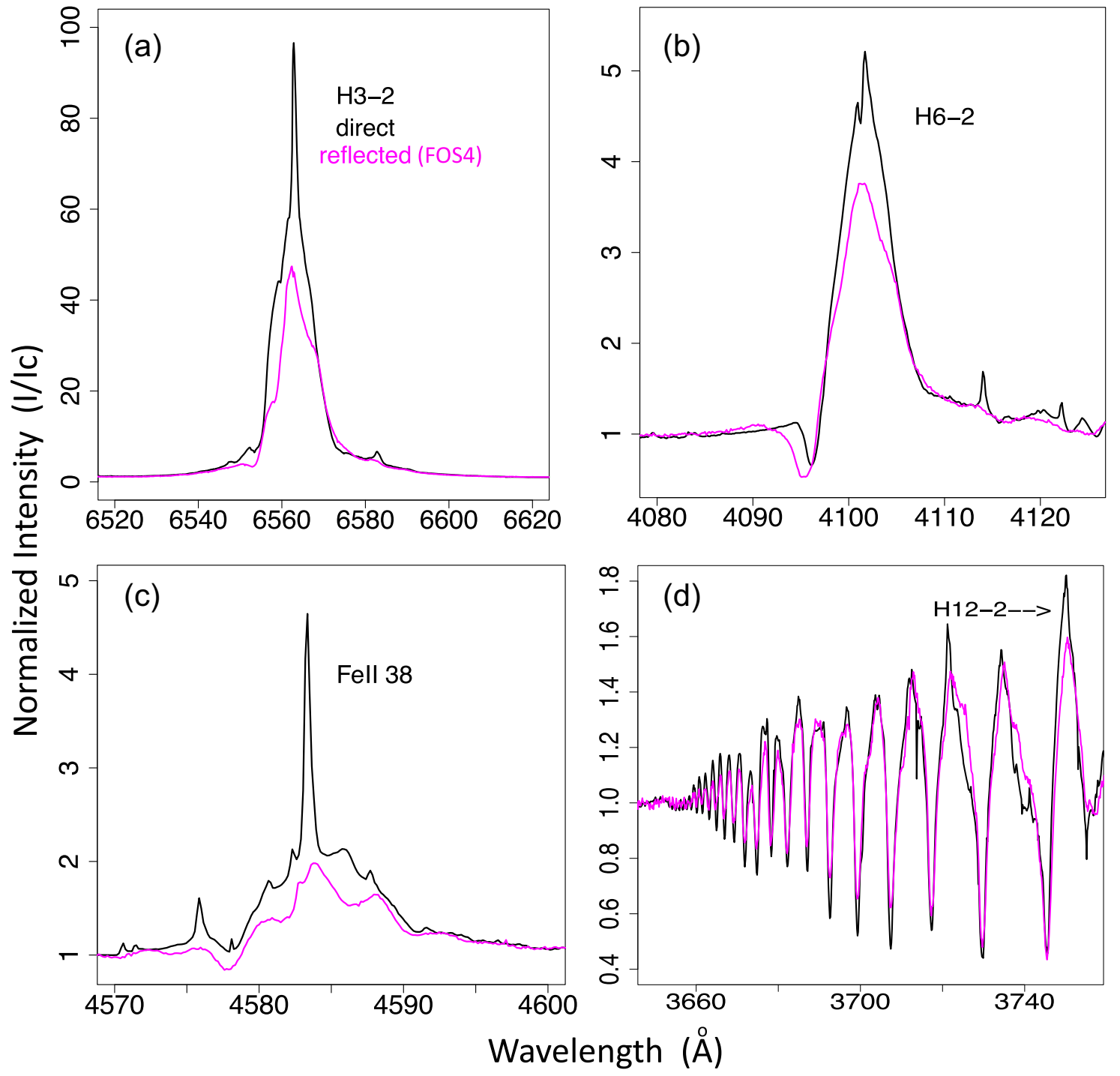

Figure 1. Profiles of representative primary wind lines in direct (black) and reflected (magenta) views. Spectra taken at high excitation phase $(\phi=11.52,2006$ May/June) with UVES. Panel (a) $\mathrm{H} \alpha$; (b) $\mathrm{H} \delta$; (c) Fe II $\lambda 4585 \AA$; (d) High members of the Balmer series. Radial velocity shifts have been applied in order to match the centroid positions of the emission lines in the reflected spectrum with those in direct light.

(vi) Optical spectra were taken at the Coude focus of the $1.6-m$ telescope at the Pico dos Dias Observatory (OPD/LNA-Br) In the optical range a variety of CCDs were used, covering $\sim 500$ to $\sim 1000$ $\AA$ at each grating angle. The response of the system as a function of wavelength is very flat and a simple linear fit can be used to rectify the stellar continuum in each spectral range, in contrast to the blaze function of the Echelle spectrographs. The room temperature of the spectrograph is very stable, resulting in accurate radial velocities. In the period 1989-2005 we used thick CCDs, delivering $\mathrm{R} \sim 6000$. After the year 2005 we used $2048 \times 2048$ thinned CCDs delivering $\mathrm{R} \sim 12000$. The typical slit width was $\sim 1.5 \operatorname{arcsec}$ and the seeing varied from $\sim 1.0$ to $\sim 3.0$ arcsec, although a number of observations were done at higher airmasses, which implies somewhat worse seeing.

(vii) NIR spectra were taken at the Coude focus of the 1.6-m telescope at the Pico Dos Dias Observatory (OPD/LNA-Br) to survey the He I $10830 \AA$ line since 1989 . Before 2001 we used thick CCDs delivering $R \sim 12000$, covering the spectral range $\lambda 10700-11000$ $\AA$. Although having efficiency lower than 1 percent, these arrays did not exhibit noticeable fringes like the thinned CCDs, which were used after 2005. The thinned CCDs delivered $R \sim 22000$ and covered approximately the same spectral range as the thick ones. In the period 2001-2004 a HgCdTe infrared array was used, delivering $R \sim 7000$ (Groh, Damineli \& Jablonski 2007), covering the spectral range 210 450-11000 A.

(viii) A NIR spectrum taken at CRIRES/VLT/ESO-Ch on 2009 April 3 and reported by Groh et al. (2007) was used to explore the spectral absorption feature at $\lambda 10792 \AA$. The resolving power was $R=90000$ and spatial resolution 0.33 ).

\section{RESULTS}

\subsection{Spatially resolved line strengths}

We checked the finding by Hillier \& Allen (1992) who reported that spectral lines scattered by the Homunculus suffer less extinction than those observed in direct light towards the central star. UVES observations were carried out with sub-arcsecond spatial resolution, 
not requiring complicated correction for the light scattered inside the spectrograph as in the previous work. Fig. 1 shows representative lines we used.

A basic characteristic of the wind lines is that the higher the upper energy level, the closer the line originates to the primary's stellar core. Lines of lower excitation arise from increasingly larger regions of the primary's wind (which dominates the wind emission), while higher excitation wind lines originate in the inner wind near the star. Thus, the comparison of continuum-normalized wind line-profiles of different excitation towards the star and reflected by the Homunculus can be used to map the spatial extent of the wind.

Panels (a) through (c) in Fig. 1 display Balmer lines by increasing upper level. The similarity between the direct and reflected light improves from lower-level transitions [e.g. $\mathrm{H} \alpha$ and $\mathrm{H} \delta$, Figs 1(a) and (b)] towards upper-level transitions (Fig. 1c). We use here model parameters for the central star using CMFGEN, quoted in Section 4.1. The $\mathrm{H} \alpha$ line (Fig. 1a) forms up to large radii in the stellar wind $\left(r \sim 100 \mathrm{R}_{*}=12000 R_{\odot}=56 \mathrm{au}=24\right.$ mas $)$. Therefore its wind component is mostly insensitive to LOS absorption, contrary to the continuum flux, which increases towards the star. Thus the apparent decrease in the continuum-normalized strength of the $\mathrm{H} \alpha$ line in the reflected spectrum (Fig. 1a) shows that the continuum suffers higher extinction in the LOS than in other directions. On the other hand, the $\mathrm{H} \delta$ line arises from a smaller region around the star and therefore is sensitive to the same small-scale LOS absorption that also affects the stellar continuum. Thus the temporal continuum-normalized $\mathrm{H} \delta$ line in the direct spectrum is more similar to that reflected at FOS4 than the case of $\mathrm{H} \alpha$ Fig. 1b).

In direct light, the upper-level transitions of the Balmer series (Fig. 1c) are very similar to those observed in the reflected spectrum, which indicates that their formation region is entirely obscured by the coronagraph. The transition H12 - 2 at the right of Fig. 1(c) is formed at $r \sim 10 \mathrm{R}_{*}$, which poses a minimum radius for the coronagraph, arbitrarily assuming it to be circular. The line Si II 6347 A displayed in panel (f) behaves very similarly to the transitions close to the limit of the Balmer series. Fig. 1(d) shows the line profiles of permitted and forbidden singly-ionized iron around $\lambda 4240 \AA$. They are typically formed at $r \sim 200 \mathrm{R}_{*}$, and suffer less extinction by the coronagraph than the stellar continuum, similar to $\mathrm{H} \alpha$. An extreme example of the contrast effect is the fact that the He II $4686 \AA$ EW is repeatable from cycle to cycle, as reported by Navarete et al. (2020) and by Teodoro et al. (2016). At periastron, the bulk of this Helium line is formed in a small region around the wind-wind collision (WWC) apex, unresolved in HST/STIS spectra. Like the upper-level transitions of the Balmer series, its characteristic disc is smaller than the coronagraph, since He II $4686 \AA \mathrm{EW}$ does not show the varying contrast effect between the stellar continuum and line emission. The He I $7065 \AA$ line displayed in panel e behaves differently from the others we have described. Its P Cygni absorption is stronger in direct than in reflected light because it has a contribution from the WWC walls (Richardson et al. 2016).

The strong, narrow emission lines superimposed on the direct stellar spectra are produced by the Weigelt knots and other fainter dense structures spatially resolved but in close vicinity to $\eta \mathrm{Car}$ (Davidson et al. 1995; Gull et al. 2016). They also appear in the spectra scattered by dust in the Homunculus, but are greatly attenuated and significantly broadened as they are intrinsically very faint. Their greatly decreased contribution in reflected light is another indication that the stellar continuum along our LOS to the primary core is largely suppressed.

Some features shown in these spectral lines may not be related to the depression of the stellar continuum, and will not be further discussed. For example, the distortion on the line profiles, like the PCygni absorption, and the shoulder in the red arm of $\mathrm{H} \alpha \lambda 6563$ $\AA$ and Fe II lines, is likely due to a latitude-dependent stellar wind (Smith et al. 2003), or the effects of the wind cavity (Groh et al. ), and inherent broadening due to velocity dispersion of the scattering dust.

\subsection{Cycle-to-cycle weakening of the EW of the primary's wind lines}

We demonstrate in Fig. 2 how the continuum level has changed relative to line profiles with two typical, singly-ionized iron lines $\lambda \lambda 5159$ and $5169 \AA$. Note that the first transition is permitted and the second is forbidden. They have been observed at nearly the same phase over four orbital cycles. This figure shows line profiles observed at $9 \mathrm{~d}$ after phase zero, with phase $\phi=9.004$ corresponding to 1992 June 20. Fig. 2(a) shows the rectified spectra for a region containing both a permitted and a forbidden transition. Both lines exhibit a broad component formed in the wind, and a narrow component that is formed in the BCD Weigelt knots located at $>0$ '.25 to the NE of the stellar core. As shown in this plot, the line's absolute EWs have decreased with time.

Fig. 2(b) shows these two lines' flux corrected using the $V$ band coeval magnitudes of the Homunculus (in the upper right) and scaled to unity at phase $\phi=9.004$ when the brightness was $V=5.64$ mag. The flux of the narrow-line components remained constant, confirming that the Weigelt knots remained at a constant brightness. This demonstrates the complementarity of ground-based observations to STIS/HST resolved spectra, as the space-based observations were the first to reveal the roughly constant brightness of the Weigelt knots (Hillier et al. 2006; Gull et al. 2009; Mehner et al. 2014).

Fig. 2(c) shows flux-normalized line profiles, which is the same as Fig. 2(b) when it is normalized to unity at the stellar continuum. This plot shows that the broad line components formed in the wind are little affected by the brightening. The broad lines have changed only slightly with time and, in particular, there is no indication for a change in the speed of $\eta$ Car's wind. Since the Weigelt knots were not affected by the changes in the continuum flux, the narrow line components in Fig. 2(c) are off-scale.

The natural explanation for these features is that the major change with time is caused by decreasing extinction due to dissipation of dust and/or tangential motion of the obscuring material covering the stellar core and possibly the extended structure located to the SE, opposite the positions of Weigelt $\mathrm{C}$ and $\mathrm{D}$ relative to $\eta \mathrm{Car}$.

\subsection{Differential weakening of Balmer lines for the central core}

As we have extensively monitored the star, especially across periastron passages since 1992 (periastron9), we can compare the evolution of line profiles at the same binary phases. Spectra recorded at phase $\phi \approx 0.025$ ( $\sim 50 \mathrm{~d}$ after phase zero) have been taken when the secondary star is deeply embedded in the extended primary wind as it passes behind the primary star. Thus, we are able to probe the relatively undisturbed wind of the primary star for a brief time (days to a few weeks) across each periastron event.

Fig. 3(a) shows the evolution of the normalized $\mathrm{H} \alpha$ line-profile across six periastron events. The strongly continuum-emitting core has become much more visible, causing the absolute EW of $\mathrm{H} \alpha$ to decrease. By contrast the $\mathrm{H} \delta$ line shows only minor changes in the emission profile (Fig. 3b), indicating that it is formed primarily behind the coronagraph. Furthermore, the blueshifted PCygni 

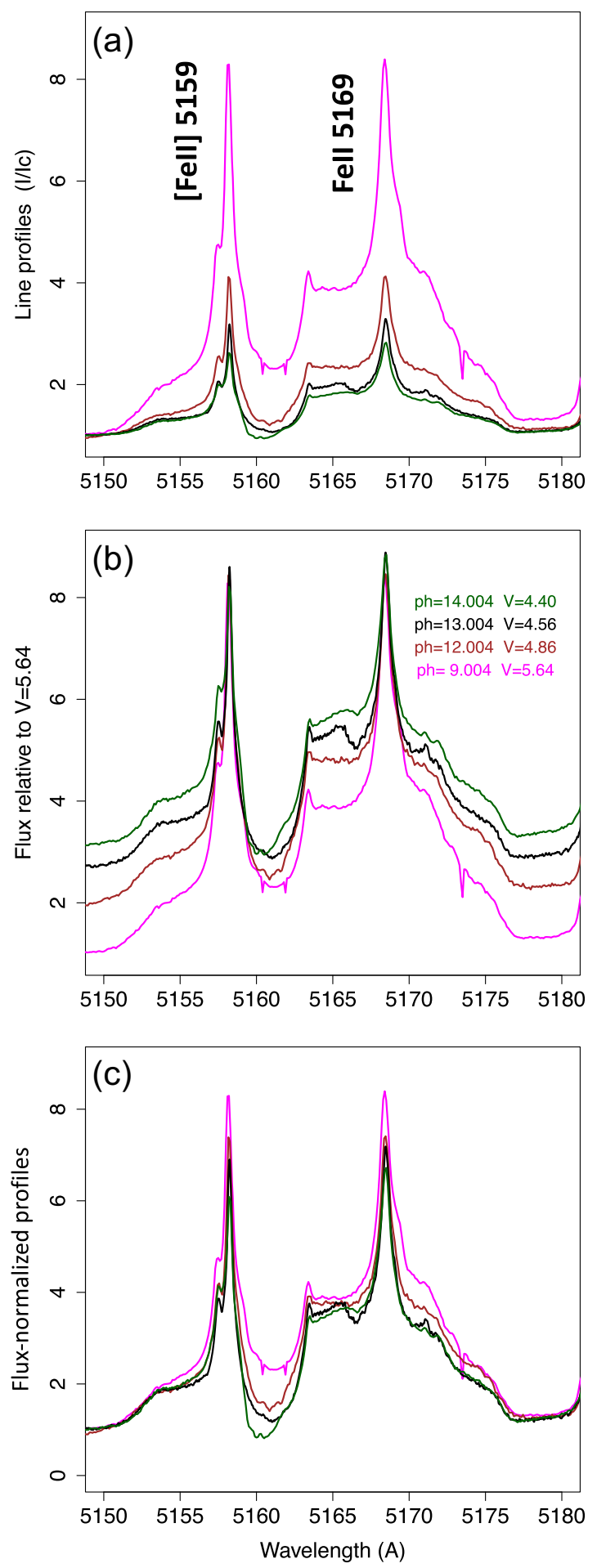

Figure 2. Variation of Fe II $\lambda 5169$ and [Fe II] $\lambda 5159$ narrow and broad components at the same phase for four orbital cycles. $\phi=9.004$ corresponds to 1992 June 20. (a) Line profiles normalized to the stellar continuum showing the long-term decreasing strength caused by the secular brightening of the stellar continuum. (b) Line profile fluxes relative to the $\phi=9.004$ brightness $(V=5.64 \mathrm{mag})$. The narrow line components remained constant because they are formed in the Weigelt knots, outside the coronagraph. (c) Fluxnormalized line profiles. The broad line components, formed in the wind, are little affected by the brightening.
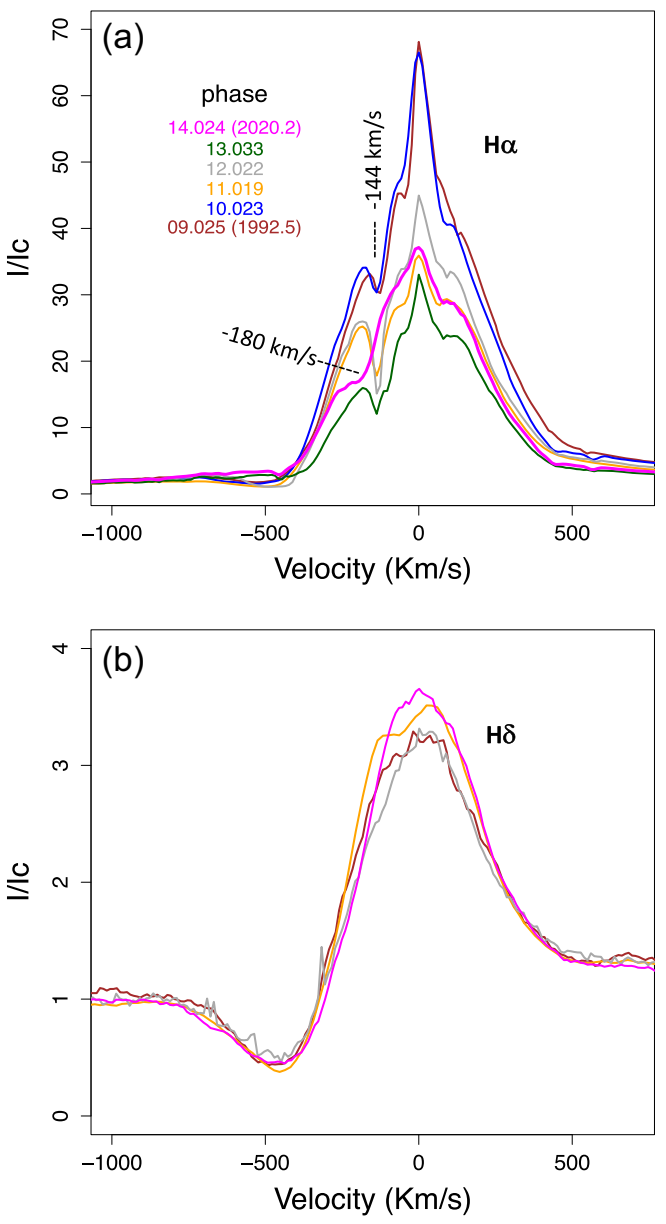

Figure 3. Evolution of $\mathrm{H} \alpha$ and $\mathrm{H} \delta$ at periastron. Line profiles of $\mathrm{H} \alpha$ (panel a) and $\mathrm{H} \delta$ (panel b) during the last six orbital cycles at the same phase $\phi \approx 0.025$ ( $\approx 50 \mathrm{~d}$ after periastron). The $\mathrm{H} \alpha$ line peak decreased by $\approx 50$ per cent while $\mathrm{H} \delta$ remained nearly constant. The constancy of the $\mathrm{H} \delta$ PCygni absorption depth, terminal velocity, and emission indicate a stable mass-loss rate. There are no $\mathrm{H} \delta$ observations at this phase for cycles \#10 and \#13. The same colours in both panels correspond the same observing dates.

absorption, which is produced along our LOS to the star, also has not changed. The constancy of the profile indicates that the terminal velocity, the mass loss, and the luminosity (by inference) of the star, have not changed very much over the last $30 \mathrm{yr}$.

\subsection{Evolution of line strengths in direct and reflected light}

Fig. 4 shows the time evolution of three representative lines of the primary's wind from 1990 to 2021: Fe II $4585 \AA$ in the top panel, $\mathrm{H} \alpha$ in the middle, and $\mathrm{H} \delta$ at the bottom. The EW absolute value ${ }^{2}$ has decreased in some cases and remained constant in others. To aid in the interpretation of the variations we fitted the data (using least squares) to a simple linear function of the form $E W=a(t-2000)$ $+b$, and the coefficients of the fits are provided in Table 1 .

Tables A3, A4, and A5 show all measurements for the star from all observatories. EWs were measured for the entire set of spectra using the same parameters as those defined by Mehner et al. (2012) for the continuum normalization and $\mathrm{EW}$ integration. The integration range

${ }^{2}$ The absolute value is used because the classical definition of EW is negative for emission lines. 

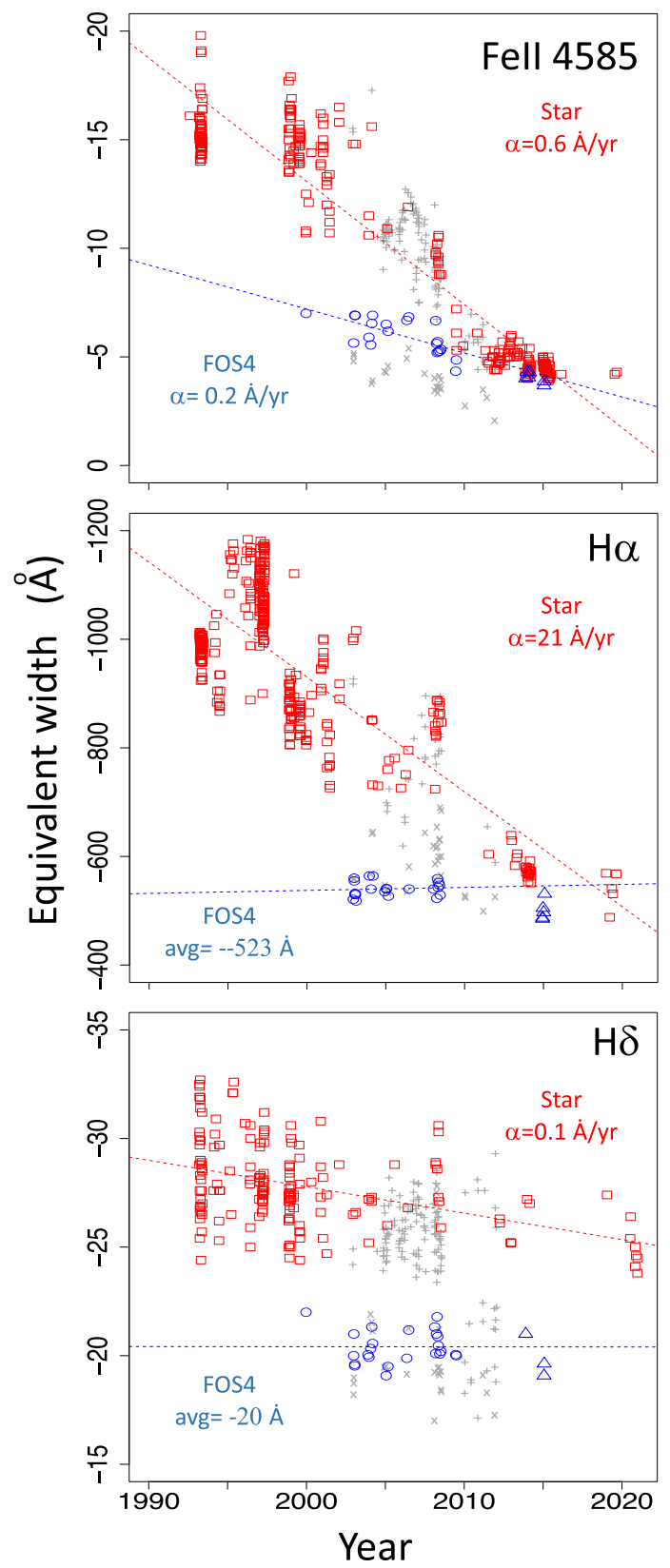

Figure 4. Cycle-to-cycle evolution of the EW of representative primary-star wind lines in direct (red) and reflected view at FOS4 (blue). Top panel: Fe II $4585 \AA$ has the steepest gradient of growing fainter in both views. Middle panel: $\mathrm{H} \alpha$ direct shows an intermediate decrease rate of the absolute $\mathrm{EW}$ and constant level $\sim-523 \AA$ at FOS4. Bottom panel: $\mathrm{H} \delta$ in direct view shows a very gentle slope and at FOS4 the line is almost constant at EW $\sim-20 \AA$. Grey points indicate measurements reported by Mehner et al. (2012), with + indicating direct view and $\times$ reflected at FOS4. Measurements in the range of phases $0.93-1.07$ were omitted in order to highlight cycle-to-cycle variations. Blue triangles indicate EWs for recent years when the telescope pointing was too far from FOS4; these were not used in the linear regression fits.

was $\lambda$ 6520-6620 $\AA$ for $\mathrm{H} \alpha, \lambda 4085-4115$ for $\mathrm{H} \delta$, and $\lambda 4570-4600$ $\AA$ for the Fe II $\lambda 4585 \AA$ line.

To place lines of different strengths on a more equal footing we use the EW ratio between the years 1995 and 2020 (R95/20 in column 4 of Table 1) to compare variations. The Fe II $4585 \AA$ absolute EW decreases at a pace $\sim 6$ times faster than $\mathrm{H} \alpha$ and $\sim 11$ times faster than
Table 1. Absolute EW rate of decrease compared to the formation radius for spectral lines in direct view to the star.

\begin{tabular}{lcccc}
\hline $\begin{array}{l}\text { Feature } \\
\text { Units }\end{array}$ & $\begin{array}{c}\text { Slope }^{a} \\
\AA^{-1}\end{array}$ & $\begin{array}{c}\text { Intercept }^{a} \\
\AA\end{array}$ & $\mathrm{R} 95 / 20^{b}$ & $\begin{array}{c}\log (\mathrm{r} / \mathrm{R} *)^{c} \\
-\end{array}$ \\
\hline $\mathrm{H} \delta$ & 0.12 & -27.5 & 1.1 & 1.5 \\
$\mathrm{H} \alpha$ & 21.13 & -990.4 & 1.9 & 1.9 \\
Fe II 4585 & 0.581 & -12.9 & 12.3 & 2.3 \\
\hline
\end{tabular}

${ }^{a}$ Linear fit EW $(\AA)=$ a. $($ year -2000$)+$ b

${ }^{b} \mathrm{R} 95 / 20=\mathrm{EW}(1995) / \mathrm{EW}(2020)$ using the linear fit

${ }^{c}$ CMFGEN model described in Section 4.1

$\mathrm{H} \delta$. The far smaller changes in $\mathrm{H} \delta \mathrm{EW}$ (which is formed close to the star) compared to that of $\mathrm{H} \alpha$ or Fe II $4585 \AA$ (formed far in the wind) indicates that $\mathrm{H} \delta$ is being emitted in a region that is mostly covered by the coronagraph. It also suggests that across the continuum and $\mathrm{H} \delta$ formation regions the occulter is fairly uniform. However, it does not need to be concentric to the star.

Table A6 contains EWs of the same three lines reflected at FOS4. At this position, the decreasing rate was $-0.2 \AA \mathrm{yr}^{-1}$ for the absolute EW of Fe II $4585 \AA$; the $\mathrm{H} \alpha$ line strength remained constant at -523 $\AA$; and $\mathrm{H} \delta$ also remained constant at $-20 \AA$. The blue triangles at the right end of the FOS4 series were extracted from spectra centered at distances larger than 0.5 arcsec from FOS4. Although they follow the series reasonably well, were not used in the calculations.

Changes in the $\mathrm{H} \alpha \mathrm{EW}$ measured in direct light seem to have stalled during the last orbital cycle (after 2014.5), with a value approaching the EW of the Homunculus-reflected light. This is expected when the coronagraph has completely vanished. But this is earlier than predicted by Damineli et al. (2019) and may be due to a temporary halt in the dissipation/shifting of the coronagraph.

\subsection{The blue-displaced absorption (shell-like feature) in the $\mathrm{H} \alpha$ line profile}

The blue absorption feature in the $\mathrm{H} \alpha$ line profile [see Fig. 3(a)] is a signature of material in our LOS, which might be related to the coronagraph. It was first reported by Ruiz, Melnick \& Ortiz (1984) and detected periodically in all subsequent low excitation events that occur near inferior conjunction of the primary (Damineli et al. 1998; Richardson et al. 2010; Richardson et al. 2015). It is not intrinsic to the primary wind; however, its velocity is consistent with that of the Little Homunculus (LH) nebula (Ishibashi et al. 2003; Gull, Kober \& Nielsen 2006). When the system is outside periastron, the LH outside the LOS is fully ionized by the FUV emitted by the secondary star and the WWC and escaping through the shock hole. However, the ionized hydrogen recombines when the dense wind of the primary inhibits the escape of the photons from the secondary, but allows Lyman $\alpha$ to scatter in the circumstellar medium (Johansson et al. 2005). This occurs when there is a neutral or partly ionized intervening gas in our LOS, which produces a typical shell-like absorption, similar to that observed in some Be stars. The shell-like feature which has $\mathrm{RV}=-144 \mathrm{~km} \mathrm{~s}^{-1}, \mathrm{FWHM}=25 \mathrm{~km} \mathrm{~s}^{-1}$, was replaced by a broader component in cycle \#14, with FWHM $=82 \mathrm{~km} \mathrm{~s}^{-1}$ centered at RV = $-180 \mathrm{~km} \mathrm{~s}^{-1}$, which continues to be compatible with the LH velocity. This indicates that the primary's wind, or the region of the LH causing the shell-absorption, suffered a change during the last periastron.

Fig. 5 presents the EW historic time series of the shell-like feature associated with $\mathrm{H} \alpha$. The dashed lines indicate phase zero of the periodic events. In addition to the periodic component, there are high-intensity excursions of the EW after phase zero of cycles \#09, $\# 10$, and \#11. These mid-cycle peaks have duration of $4 \mathrm{yr}$ or less 


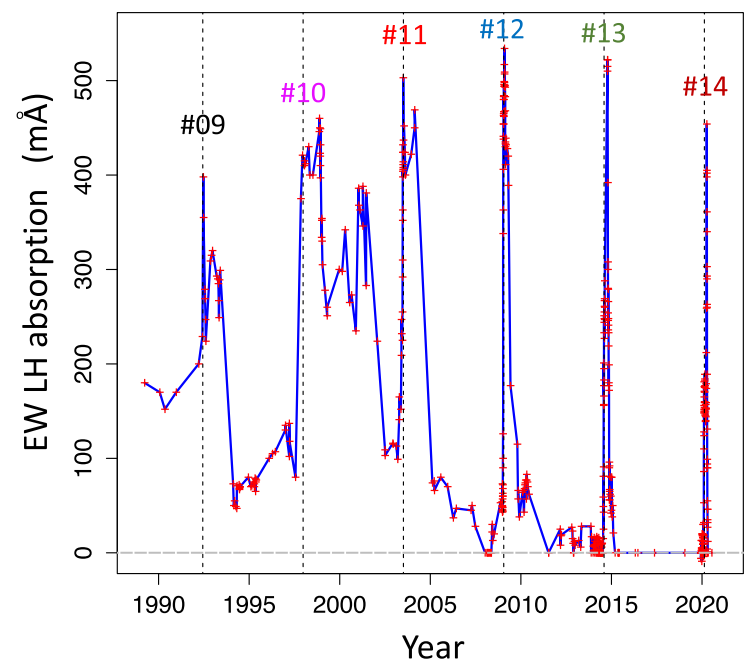

Figure 5. Historical EW time series of the blue displaced absorption on the $\mathrm{H} \alpha$ line profile - originated in the $\mathrm{LH}$ - showing three components: (a) the periodic peak at periastron, (b) occasional excursions to high intensity after periastron, and (c) a relatively low-intensity component always present, but fading in the long term. Labels \#09 to \#14 indicate the orbital cycles. The EW was measured by direct integration over the $\mathrm{H} \alpha$ line profile, in the range $\lambda$ 6548-6556 ̊.

and might be due to dense structures passing across our LOS to the secondary star. They appear to have not been present in cycle \#13. The third feature in this time series is a low-level absorption component that was present outside periastron and has been decreasing in EW with time. It remained at $\mathrm{EW} \sim 0$ after the low excitation event \#13.

The periodic peaks of the blue $\mathrm{H} \alpha$ absorption EWs (Fig. 5) are not produced by the coronagraph, which varies on longer time-scales. The occulter that causes the periodic peaks around periastron is the primary's wind, produced when the secondary star moves behind the primary near periastron. The shell-like absorption is also observed outside periastron and has a long-term behaviour. It is probably produced by other regions around the binary system. The long-term variable shell-like absorption is produced by the coronagraph. The complex behaviour of the shell-like absorption indicates that there are other occulters located in the circumstellar medium, located farther from the primary star but closer than the LH.

A similar narrow absorption line also exists in other directions, as reported by Boumis et al. (1998). That direction could cross denser circumstellar material inside the LH that is sampled by our LOS and must be permanently neutral or partly ionized. This means that the UV radiation emitted through the WWC cavity never illuminates that direction. That shell-like absorption is reflected on a small nebula at $>2$ arcmin to the NW of $\eta$ Car, outside the Homunculus, and is seated at $\mathrm{RV}=-80 \mathrm{~km} \mathrm{~s}^{-1}$ on the $\mathrm{H} \alpha$ line profile. It is stronger than its sibling along our LOS and has not varied during 1985-1997. We took one spectrum with the Goodman/SOAR spectrograph in 2018 at the same spot as observed by Boumis et al. (1998). Although our spectra have lower spectral resolution, no major changes were seen. This seems to indicate that the shell-like feature sampled in our LOS is part of a much larger structure.

Pickett et al. (2021) reported a similar decrease of the EW weakening of the $-144 \mathrm{~km} \mathrm{~s}^{-1}$ narrow absorption component of $\mathrm{NaD}$. Based on STIS/HST UV spectroscopy, Gull et al. (in preparation) noted similar disappearance of narrow absorption lines. The $H S T / \mathrm{STIS}$ spectra also show that the ionization is increasing not only in our LOS, but also within the SW Homunculus lobe, changing

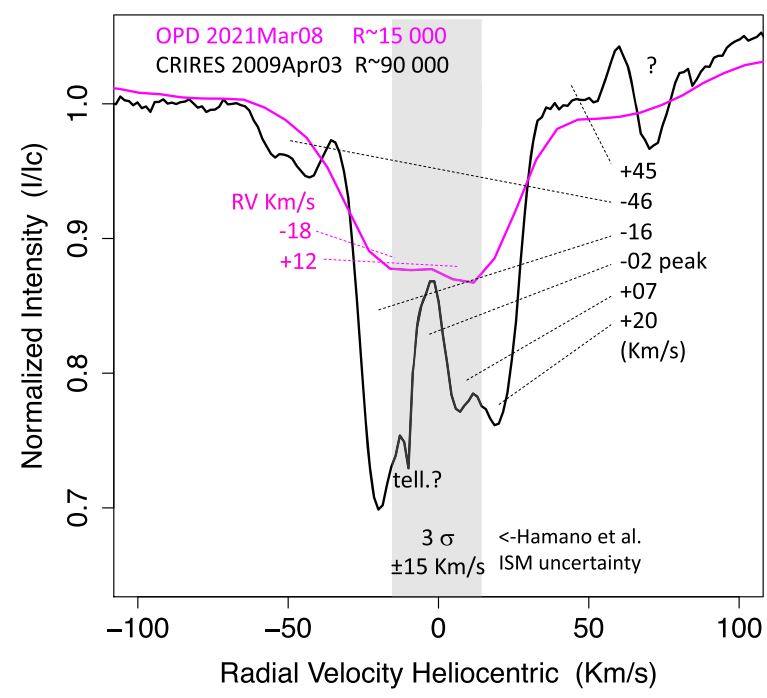

Figure 6. Profile of the absorption line at $\lambda 10792 \AA$. The black line is from a CRIRES spectrum taken on 2009 April 03, showing at least four components blueshifted and redshifted between $\pm 45 \mathrm{~km} \mathrm{~s}^{-1}$ from the central reversal. Zero velocity corresponds to the position measured by Hamano et al. (2015) for a diffuse interstellar band at the same position and the grey strip marks $3 \sigma$ uncertainty in their measurements. The magenta line shows an observation at OPD/Coudé on 2021 March 08, with four times lower spectral resolution. It seems that the two main components of the absorption line changed the intensity ratio between 2009 and 2021.

dramatically to the NW of the stellar core. This indicates that the coronagraph may be extended, with its edge projected against the LH. The vanishing occulter modulating the FUV must be within the $\mathrm{LH}$. The remaining occulting material in the coronagraph also affects recombination lines, but at larger distances, beyond the $\mathrm{LH}$.

\subsection{A circumstellar diffuse interstellar band at $\lambda 10792 \AA$ ?}

We have explored the possibility that the isolated absorption feature at $\lambda 10792 \AA$, hereafter eta1.079, is a circumstellar counterpart of a diffuse interstellar band (DIB) (Díaz-Luis et al. 2015). In addition to the agreement in wavelength position, its long-term strength decrease is well correlated with the decreasing extinction, and it has a gradient opposite to the P Cygni absorption strength of He I $10830 \AA$ and most of the resonant lines in the optical range.

This feature was reported in $\eta$ Car by Damineli Neto et al. (1993) who attributed it to a blue displaced absorption at RV = $-1040 \mathrm{~km} \mathrm{~s}^{-1}$ associated with the He I $10830 \AA$ Aine as also adopted by Groh et al. (2010). However, no similar feature at this velocity was present in the He I $20587 \AA$ 'sister' line shown in that work and any other $\eta$ Car spectral line reported so far.

In order to examine its profile and wavelength, we plot a highresolution spectrum reported by Groh et al. (2010) in Fig. 6, taken on 2009 April 03. Two other CRIRES spectra (from 2008 May 05 and 2009 Feb 09) reported in that work show a similar line profile close to $\lambda 10792 \AA$ except for a central peak above the continuum, which might be a defect, not necessarily a real emission. The absorption feature at this wavelength shows a complex profile, with at least four components between +45 and $-46 \mathrm{~km} \mathrm{~s}^{-1}$. There is a central reversal feature in almost exact coincidence with the $\lambda 10792$ DIB reported by Hamano et al. (2015). The vertical grey strip in Fig. 6 shows the $3 \sigma$ uncertainty around $\lambda 10792.15 \pm 0.15 \AA$ reported by 


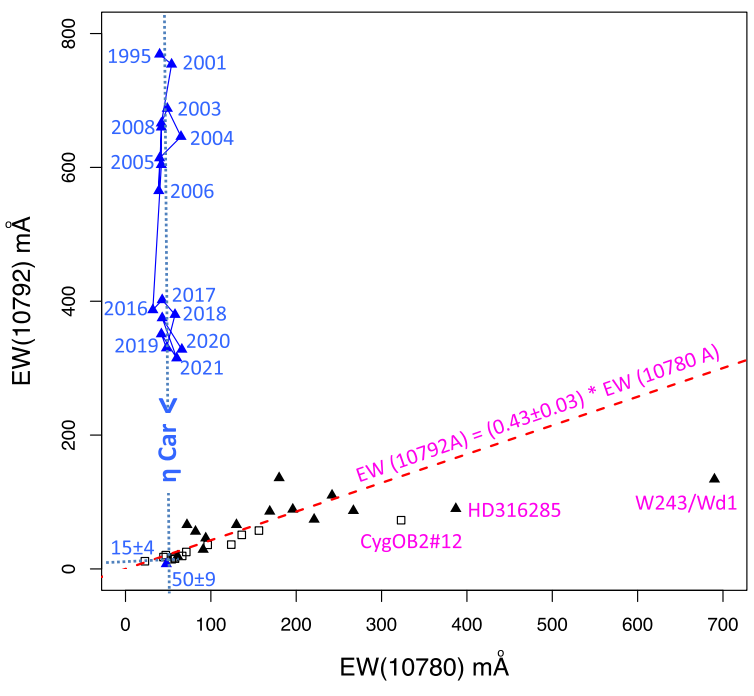

Figure 7. DIBs at $\lambda 10780 \AA$ (DIB1) and $\lambda 10792 \AA$ (DIB2) in a sample of stars. Squares are from Hamano et al. (2015) and triangles from this work. Points labelled in magenta indicate deviating objects from the linear relation between the two DIBs: $\mathrm{EW}(10792 \AA)=(0.43 \pm 0.03) \mathrm{EW}(10780 \AA)$. Blue triangles indicate a long-term fading of the $\lambda 10792 \AA$ absorption in $\eta$ Car (here called eta1.079).

Hamano et al. (2015) for many directions in the Galaxy - hereafter named DIB2.

A recent observation taken at the OPD/Coudé focus on 2021 March 08 with $\mathrm{R}=15000$, four times lower than CRIRES, is also shown in Fig. 6. The low amplitude reversal is at the same velocity as in CRIRES. The two main absorption components are shown at approximately the same velocity, but with interchanged depth ratio, in addition to this variation, the EW has been decreasing slowly over the long term, as described below.

Although the large positive velocity makes the identification of this feature as DIB2 unlikely, in order to gain more information, we measured the DIB2 on many normal and emission-line stars with spectra taken at the OPD/Coudé (Table A1). Fig. 7 shows our measurements of DIB2 compared with the closest one at $\lambda 10780$ $\AA$ (hereafter DIB1). Fig. 7 shows a very good linear correlation for most of the observations of ours and of Hamano et al. (2015): $\mathrm{EW}(\mathrm{DIB} 2) / \mathrm{EW}(\mathrm{DIB} 1)=0.43 \pm 0.03$. These authors found a single discrepancy of the correlation for CygOB2\#12 and we show two additional exceptions for the LBVs HD 316285 and W243 in the Westerlund 1 cluster. These measurements are based on sufficiently large EWs, making them quite reliable. These three objects have much larger extinction and larger DIB1 EW, but have their DIB2 EW much fainter than expected. This indicates that DIB1 and DIB2 only mimic belonging to the same family of carrier molecules when measured in the solar vicinity but might have different behaviour in other parts of the Galaxy.

Fig. 7 includes measurements of eta1.079 since 1995 (Table A2). The feature eta1.079 is exceptionally strong compared to DIB1. When comparing its large EW with CygOB\#12, HD 316285 , and W243, which deviate in the opposite sense with respect to the linear fit, it seems unlikely that eta1.709 is produced by the same carriers as DIB1. However, there are only a few cases studied at very high reddening and we cannot reach a firm conclusion. Despite the relatively large errors in EW, the long-term behaviour of this feature in $\eta$ Car is clear: its intensity is decreasing with time. On the other hand, the strength of DIB1 is consistent with constant EW. eta1.079

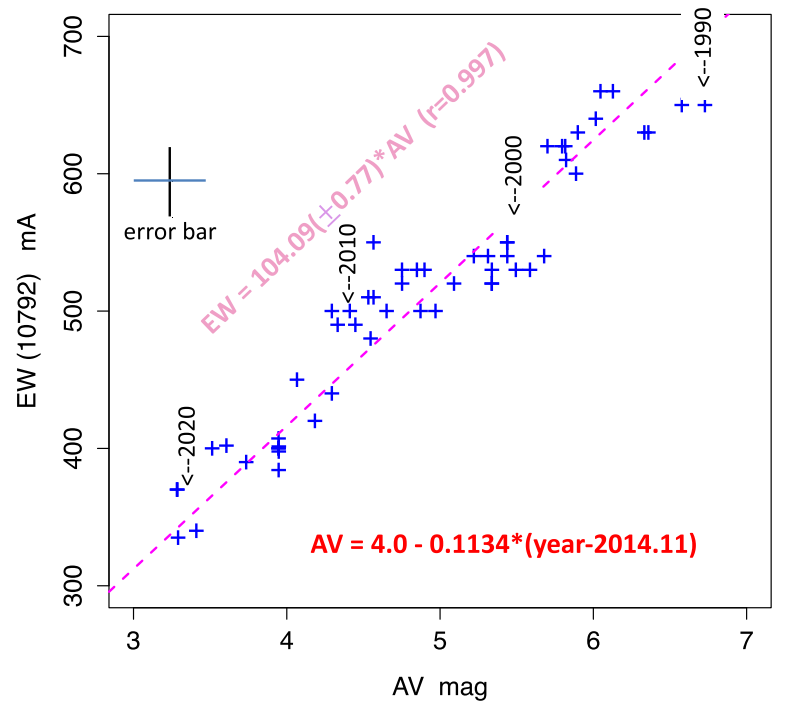

Figure 8. Relation between eta1.079 ( $\lambda 10792 \AA$ absorption) and $A_{\mathrm{V}}$. The extinction towards the stellar core ( $x$-axis) was reported by Damineli et al. (2019). It is fading in the form $A_{\mathrm{V}}(\mathrm{mag})=4.0-0.113$ (year -2014.11 ) and is predicted to reach the interstellar medium (ISM) value $A_{\mathrm{V}} \sim 1.5-$ $2.4 \mathrm{mag}$ in 2028-2036. The EW of eta1.079 absorption ( $y$-axis) is fading in tight correlation $(r=0.997)$ with the visual extinction of the central star: $\mathrm{EW}(\mathrm{DIB} 2)(\mathrm{m} \AA)=104.09 A_{\mathrm{V}}$. The typical error bar of the individual measurements is shown as a cross in the top left of the plot (see Table A2).

has been decreasing at a rate of $\sim-10 \mathrm{~m} \AA \mathrm{yr}^{-1}$ that evolves on the contrary sense to the P Cyg absorption in many spectral lines. For example, the P Cyg absorption of He I $10830 \AA$ in the same period increased at a rate $\sim+500 \mathrm{~m} \AA \mathrm{yr}^{-1}$. If eta1.079 is not produced by DIB1 carrier molecules, it might be due to another mixture of molecules and does not seem to be an absorption associated with the primary wind.

\subsection{Correlated decrease of $A_{V}$ extinction and the eta1.079 absorption within the coronagraph}

Damineli et al. (2019) showed that the rate of decrease in extinction follows the equation $A_{\mathrm{V}}(\mathrm{mag})=4.0-0.113$ (year -2014.11$)$. If the excess absorption of eta1.079 in our LOS to the central star originates in the coronagraph, it would follow the extinction decrease. We used the above relation to derive $A_{\mathrm{V}}$ for the dates we have measured EW(eta1.079) and found a surprisingly tight correlation: $\mathrm{EW}(\mathrm{eta1} .079)(\mathrm{m} \AA)=104.09 A_{\mathrm{V}}(\mathrm{mag})$, with $\mathrm{r}>99$ per cent $($ dashed magenta line in Fig. 8). Spectra taken in the time interval 19902021 are labelled for every decade in the plot to indicate how the changes vary with time. Despite the relatively large errors in EW measurements (see Table A2), we see that the changing EW of eta1.079 follows the decreasing extinction inside the coronagraph.

The normal way to produce extinction is by dust, and so the correlation found in Fig. 8 might suggest that the eta1.079 carrier molecules are associated with dust grains or at least they are mixed inside the same region. The brightening in the $V$ band between orbital cycles 10.74 (2002.07) and 13.69 (2018.44) was $1.86 \mathrm{mag}$ as compared with 2.9 mag at $1200 \AA$ measured in STIS spectra (Damineli et al., in preparation). If the brightening was due to just decreased extinction, the increase in brightening at $1200 \AA$ should have been eight magnitudes for a canonical reddening law $\left(R_{\mathrm{V}}=\right.$ 3.1). This indicates that the extinction of the occulter was mostly 


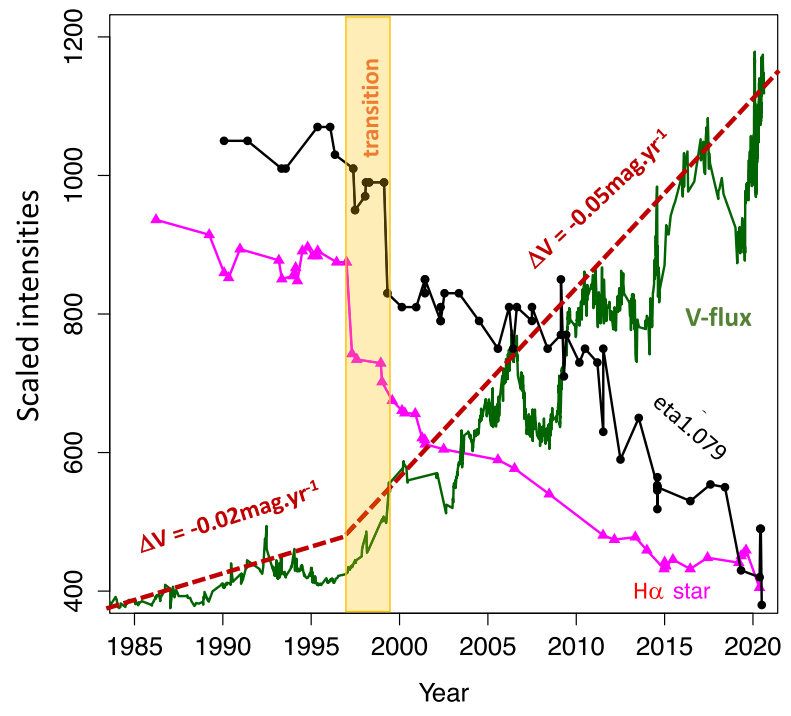

Figure 9. Structures in the coronagraph. The long-term brightening of the entire object (star + Homunculus) due to decreasing extinction towards the stellar core is shown by the green line. Also shown are the EW of eta1.079 (black line) and the absolute $\mathrm{EW}$ of $\mathrm{H} \alpha$ (magenta line), both measured in the star. A strong inverse correlation between the EWs of eta1.079 and $\mathrm{H} \alpha$ with the $V$-band flux is observed. The $V$-band light curve (green line) is based on data taken from Damineli et al. (2019) and transformed to relative flux.

grey, which requires very large dust grains or electron scattering in an ionized gas. Large dust grains are supported by dust model results of Morris et al. (2017), in particular, iron grains in the range of few to tens of microns. Such large grains would condense close to the central source at temperatures of a few hundred to $800 \mathrm{~K}$ and are not in thermal equilibrium. Such a high temperature in principle is against the identification of eta1.079 as the same carrier molecule as DIB2, which is formed in the very cold ISM.

\subsection{Spatial structures inside the coronagraph}

Fig. 9 compares $\mathrm{H} \alpha$ and eta1.079 EWs plus $V$-band flux variability from 1985 through 2020 . The $y$-axis was scaled to represent the measured absolute $\mathrm{H} \alpha \mathrm{EW}$ in $\AA$. Both the eta1.079 EW and the $V$ band flux values were multiplied by constants to fit within the plot. The fading of the eta1.079 EW (black line) occurs in concert with the $\mathrm{H} \alpha \mathrm{EW}$ (red line). The $V$-band flux of the whole object (stellar core + Homunculus, green line) increases with time. A well-known change in the brightening rate occurred in 1997-99. It seems that there was a coeval fading of $\mathrm{H} \alpha$ and eta1.079, labelled as 'transition' in Fig. 9. If this transition was as sharp as it appears to be, it would not be consistent with systematic coronagraph dissipation but implies a clumpy portion of the coronagraph moving out of our LOS.

\section{DISCUSSION}

\subsection{A model for the central star}

To facilitate the discussion we present a model for the primary star, assuming a distance of $2.3 \mathrm{kpc}$ to the object (Allen \& Hillier 1993; Meaburn, Walsh \& Wolstencroft 1993; Davidson et al. 2001; Shull, Darling \& Danforth 2021).

Following previous works (Hillier et al. 2001, 2006) we chose as basic parameters of the model:

$$
\begin{aligned}
& R_{\text {core }}=120 \mathrm{R}_{\odot} \\
& \dot{M}=8.0 \times 10^{-4} \mathrm{M}_{\odot} \mathrm{yr}^{-1} \\
& L=5.0 \times 10^{6} \mathrm{~L}_{\odot} \\
& V_{\infty}=420 \mathrm{~km} \mathrm{~s}^{-1}
\end{aligned}
$$

These are similar, but not identical to earlier values adopted by Hillier et al. (2001):

$$
\begin{aligned}
& R_{\text {core }}=60 \mathrm{R}_{\odot} \\
& \dot{M}=1.0 \times 10^{-3} \mathrm{M}_{\odot} \mathrm{yr}^{-1} \\
& L=4.0 \times 10^{6} \mathrm{~L}_{\odot} \\
& V_{\infty}=400 \mathrm{~km} \mathrm{~s}^{-1}
\end{aligned}
$$

And also similar to Groh et al. (2012a):

$$
\begin{aligned}
& R_{\text {core }}=60 \mathrm{R}_{\odot} \\
& \dot{M}=8.0 \times 10^{-4} \mathrm{M}_{\odot} \mathrm{yr}^{-1} \\
& L=5.0 \times 10^{6} \mathrm{~L}_{\odot} \\
& V_{\infty}=420 \mathrm{~km} \mathrm{~s}^{-1}
\end{aligned}
$$

The differences in the physical parameters are explained as follows. The luminosity comes from infrared measurements - its precision (for a fixed distance) is primarily limited by a correction for flux not absorbed by dust, and a correction for the luminosity of the companion star. Due to the large mass-loss rate, the primary wind is optically thick, even in the visible. The core radius cannot be well determined - possible values range from 60 to several hundred $R_{\odot}$. Larger values are preferred if we assume that the He I line emissions arise primarily in the wind-collision interface between the two stars as a consequence of the ionizing flux of the secondary star. In this work we adopt $R_{\text {core }}=120 \mathrm{R}_{\odot}$.

Models of the central star are riddled with systematic uncertainties arising from the following:

(i) Due to circumstellar extinction, we do not know the amount of reddening, nor the shape of the local extinction law. As a consequence, there is no absolute flux scale in the optical or UV.

(ii) As discussed below, the circumstellar extinction alters the spectral appearance of the star (even with HST data). As a consequence, Hillier et al. (2001) did not model the 'intrinsic' spectrum of the central star in the modelling of the HST spectrum obtained near periastron in December of 1998.

(iii) The companion star influences the observed spectrum via its ionizing radiation and the interaction of its wind with that of the primary (Groh et al. 2012a,b; Madura \& Groh 2012). At apastron, the companion star lies at a distance of $\sim 30$ au $\left(\sim 6500 \mathrm{R}_{\odot}\right)$ from the primary. The companion star carves a cavity in the primary's wind, and this cavity partially overlaps (in radius) with the line formation region in the primary wind. Its influence on the primary's spectrum is partially governed by the opening angle of the cavity, and hence the relative wind momenta of the two stars. While the secondary is believed to be less luminous than the primary (Mehner et al. 2010a), 


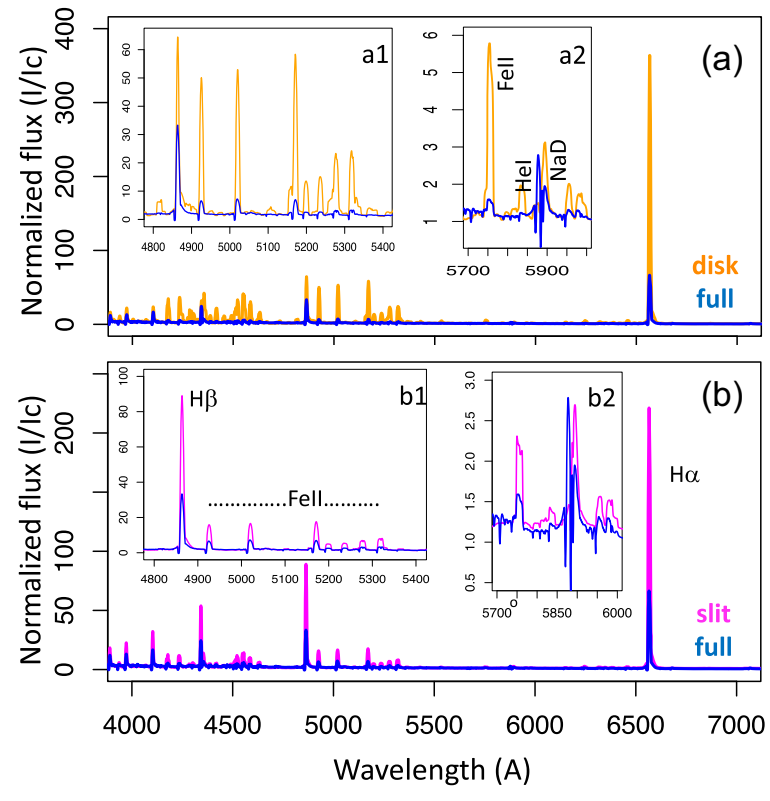

Figure 10. Spectra from CMFGEN models with and without obstruction of the central star by a coronagraph. The blue line is the spectrum of the entire object (star plus wind) Panel (a): Obstructing coronagraph as a circular disc centered on the star with radius $r=11.5$ mas (26.45 au). Panel (b): Extraction inside a slit of infinite length and 25 mas wide, in which the star is placed at 2.5 mas ( $5.75 \mathrm{au}$ ) from the slit border. Insets display zoomed views of spectral regions of interest.

its ionizing UV radiation field $(\lambda<912 \AA)$ is much stronger than that of the cooler primary.

(iv) Line emission may also arise from the wind collision zone.

(v) The wind may be asymmetric (Smith et al. 2003; van Boekel et al. 2003; Weigelt et al. 2007).

Despite these difficulties and uncertainties, the model of the primary star can still be used to glean insights into the nature of the coronagraph.

\subsection{CMFGEN studies of impact on the spectrum by different obstructing structures}

Two models were constructed with the CMFGEN model (Hillier \& Miller 1998) to assess the impact of an obstruction in front of the central star. First, we computed the unobscured optical spectrum of $\eta$ Car A as shown by a blue line in Fig. 10. The orange line in Fig. 10(a) shows the spectrum integrated outside a circular disc of radius $r=11.5$ mas, concentric to the star. In Fig. 10(b) the magenta line shows the integrated flux from inside a slit of infinite length and 25 mas wide. The slit is offset perpendicularly to its length so that the centre of the star is 2.5 mas outside the slit. This second geometry is inspired by the emission structure observed by Millour et al. (2020) and by Falcke et al. (1996) and by the spatial variability of the nebular ionization analysed by Gull (in preparation). As expected, the coronagraph enhances the wind lines relative to the unimpeded view of the spectrum in both geometries, contributing to the majority of the continuum flux. Since most of the Fe II, and particularly the [ Fe II ] emission lines are formed at larger radii than the Balmer lines, they show a greater increase in the EW absolute values. For the same reason, the intensity ratios of $\mathrm{H} \alpha$ to the upper members of the Balmer series also increase.
Table 2. EW (in $\AA$ ) of representative lines extracted from CMFGEN models and observations.

\begin{tabular}{lccccc}
\hline Feature & Full $^{c}$ & Disc $^{b}$ & Slit $^{a}$ & $1999.1^{d}$ & $2019.9^{d}$ \\
\hline $\mathrm{H} \alpha$ & -646 & -3675 & -2504 & -876 & -551 \\
$\mathrm{H} \beta$ & -131 & -355 & -444 & -139 & -121 \\
$\mathrm{H} \delta$ & -35 & -81 & -121 & -28 & -21 \\
$\mathrm{Fe}$ II 5317 & -15 & -178 & -67 & -13 & -6 \\
\hline
\end{tabular}

$\overline{\left.{ }^{a} \text { Extraction inside a slit aperture from } d=2.5 \text { to } 27.5 \text { mas (5.75 to } 63.25 \mathrm{au}\right)}$ of the central star and infinite length; ${ }^{b}$ Extraction outside a circle with $r=$ 11.5 mas (26.45 au); ${ }^{c}$ Spectrum extracted over the entire field (star + wind); ${ }^{d}$ observations taken with FEROS/ESO (1999.9) and NRES/LCOGT (2019.9) instruments.

In Fig. 10, excluding the Balmer lines, the strongest lines are from Fe II, while the weaker lines are due to [Fe II]. The He I transition at $\lambda 5876 \AA$ is much stronger in the full spectrum (blue curve) since the line forms close to the star because Helium is mainly neutral in the outer wind.

There are numerous possibilities for the geometry and size of the coronagraph, including sharp and smooth boundaries. Our intent was not to fit the observed spectrum, which demands additional constraints from the observations, but to explore the general EW trends of an occulter in front of the star and its wind spectrum.

In Table 2 we present the EW for a set of lines calculated in three CMFGEN model spectra shown in Fig. 10: the unobscured view (full); a coronagraph occulting only the stellar core (disc); and a the occulter covering the whole field of view, except for a slit, which are compared to two observed spectra: one taken with FEROS/ESO in 1999.1, when the impact of the coronagraph was still large ( $A_{\mathrm{V}} \sim 4 \mathrm{mag}$ ), and one taken with NRES/LCOGT spectrograph (with instrumental setup similar to that of FEROS/ESO) in 2019.9, when the impact of the coronagraph had decreased to $A_{\mathrm{V}} \sim 1$ mag. The decrease in line strengths between these two dates follows the same trend in the spectrum changing from that of an occulted half stellar wind (slit) to the unocculted spectrum (full). This is clearly demonstrated in Table 2 that shows EW as a function of the models (columns 2, 3, and 4) and for the different epochs (columns 5 and 6).

\subsection{Observational constraints for the position and shape of the coronagraph}

While the dusty occulter may be changing by tangential motion, irregular, clumped substructure, and long-term dissipation, we describe a simple model to constrain its size. Assume the coronagraph is circular, homogeneous, motionless, concentric with the primary star, and only its transparency is decreasing. The maximum size of the coronagraph can then be derived by using the projected distance of the Weigelt knots (Weigelt \& Ebersberger 1986) closest to the central star. While Weigelt $\mathrm{B}$ has faded, the two other clumps ( $\mathrm{C}$ and D) have remained constant in flux, at the same phase of the orbital cycle, in the 1998-2004 period, in the optical and UV windows (Gull et al. 2016). Unlike the central source, the Weigelt knots suffer very low extinction, $A_{\mathrm{V}} \sim 2.0 \pm 0.5$ mag (Teodoro et al. 2020). Since the Weigelt $C$ object was within $\approx 200$ mas (462 au) from the central source in 1995 (Weigelt \& Kraus 2012), we deduce that the radius of the coronagraph must be smaller than this, assuming it to be spherical. The coronagraph cannot be located between Weigelt $C$ and the binary system since the high-excitation spectrum of Weigelt $\mathrm{C}$ has remained constant over the last two decades. The coronagraph seems not to have the same nature as the Weigelt knot, because there are indication that it is more extended, covering the SE direction. In 
addition, the Weigelt knots are located at Homunculus equator, not to our LOS to the central binary.

An estimate of the minimum size along our LOS can be obtained from estimates of the circumstellar extinction. In the modelling by Hillier et al. (2001), the derived $V$-band circumstellar extinction was approximately five magnitudes in 1998 that agrees with Damineli et al. (2019). With the physical model of the coronograph described above, and assuming the coronagraph has infinite optical depth, we obtain for a concentric circular configuration, a minimum radius of $60 \mathrm{au}$ (26 mas). This is roughly the radius at which the electron scattering optical depth is 0.01 . Since the spectrum extracted beyond this radius shows distinct differences from that of the primary star, the coronagraph must have some transparency, and thus it must have been larger than 60 au in 1998. However, its size is not well constrained to date. The flux increase observed since 1998 could be due to either an increase in transparency, a decrease in radius, or a shift in the coronagraph location relative to our LOS.

Of course, the coronagraph does not have to be circular. Falcke et al. (1996) performed speckle polarimetry and resolved an obscuring $\sim 20 \times 60$ mas SW-NE dust bar in polarized light. Recently, Millour et al. (2020) reported adaptive optics observations of $\eta$ Car obtained with SPHERE at the VLT/ESO. The spatial resolution was 20 mas ( $\sim 7 \mathrm{au}$ ), and observations were obtained in both $\mathrm{H} \alpha$ and the nearby continuum. While difficult to interpret unambiguously, the images show structures that might be connected to the coronagraph. The stellar core is occulted by a dust lane running SW to NE and there is a bi-conical structure in the polarization map running perpendicular to it (their fig. 4). Further, the area NW of the central star is more transparent than the opposite side, consistent with markedly less extinction to Weigelt $\mathrm{C}$ and $\mathrm{D}$.

\subsection{Consequences of the disappearance of the coronagraph}

The absolute EW of the eta1.079 feature is fading at a rate of -29 $\mathrm{m \AA} \mathrm{yr}{ }^{-1}$, so it will likely disappear in $\sim 2030$. The uncertainty in this prediction is large because this feature is faint and the spectra are noisy, but it is consistent with the prediction for the coronagraph vanishing time-scale derived from the decreasing rate of the $A_{\mathrm{V}}$ extinction. Following Damineli et al. (2019; their fig. 18) this will happen in the time frame 2028-2036, depending on the foreground extinction, which is uncertain in the range $A_{\mathrm{V}}=2.4-1.5 \mathrm{mag}$.

The disappearance of the coronagraph is expected to have an impact also on the absorption lines of the spectrum components, since it will allow the light from the secondary star to ionize far-out regions of the circumstellar medium that were previously shielded from it as we have shown for the narrow $\mathrm{H} \alpha$ absorption in the $\mathrm{LH}$. The details will be evaluated only after the size and position of the coronagraph are known.

The correlation shown in Fig. 8 indicates that the eta1.079 carrier molecules are related to dust grains and both are being photo-dissociated by the intense stellar radiation field. When the coronagraph has completely vanished, the stellar core will stop increasing in brightness. The predicted magnitude of the stellar core at that time is $V \sim 2.5-3.5 \mathrm{mag}$, which is a little brighter than it was in the 1600sbf (the amount of light reflected from the Homunculus is not very relevant here, because it was absent before the GE and now is fainter than the central star). The true luminosity variation depends on how much the bolometric correction (BC) changed since then, but unfortunately there were no historical reports on the colour of the star before the GE event. Let us assume that the change in BC was modest, which implies that the intrinsic luminosity did not change significantly or might have mildly increased. Since the GE ejected
$>15 \mathrm{M}_{\odot}$ following Morris et al. (1999) and Smith et al. (2003) or $>45 \mathrm{M}_{\odot}$ following Morris et al. (2017) - using a full radiative transfer model with a far more chemically realistic dust library the luminosity of the central star should have dropped by a large amount, unless it was compensated by some process. This favours the scenario of a merger in a triple system (Portegies Zwart \& van den Heuvel 2016; Smith et al. 2018; Hirai et al. 2020) over a massive wind ejection as the mechanism for the GE, since the primary star would have been mixed internally in a merger, bringing fresh fuel to its central core.

\section{CONCLUSIONS}

This work brings together various pieces of evidence indicating the presence of an obscuring structure in front of the binary system in $\eta$ Car. Some of them were suspected as long as $30 \mathrm{yr}$ ago but now there are better observational data and modelling.

The central stellar binary ( $\eta$ Car A $+\eta$ Car B) has been brightening by many magnitudes for half a century, without major changes in the intrinsic spectrum of the primary star. This is not expected for a star which was already close to the Eddington limit at the beginning of this process. The long-term brightening has a circumstellar origin. The Homunculus reflection nebula has remained at an essentially constant brightness ( $V \sim 5.48 \mathrm{mag}$ ) while the central stellar core has brightened by several apparent magnitudes (Damineli et al. 2019).

The main conclusions of this spectroscopic study are as follows:

(i) Spectral lines formed in the stellar wind have larger absolute EW in direct than in reflected light (Fig. 1).

(ii) The absolute EW values of the lines formed in the wind have decreased systematically from cycle to cycle (Fig. 2a). However, the Weigelt knots located at a projected distance of a fraction of an arcsecond from the central star have maintained a constant brightness and ionization level (Fig. 2b).

(iii) After correcting for the secular brightening, the broad lines formed in the stellar wind exhibit a constant line profile (Fig. 2c).

(iv) Line profiles of the upper Balmer series members have been almost constant for the last $20 \mathrm{yr}$, as compared to large variations in $\mathrm{H} \alpha, \mathrm{Fe} I$, and [Fe II]. This is explained by the fact that these lines are formed at large radii in the stellar wind, as compared with the high-energy spectral lines, which are mainly formed behind the coronagraph (Fig. 3). The absolute EW value of $\mathrm{H} \alpha$ in direct light has decreased by $\approx 50$ percent from 1985 to 2014 (Fig. 4). In contrast, the EW of $\mathrm{H} \alpha$ observed in the FOS4 reflected light remained roughly constant over the time interval 2000-2010. The $\mathrm{H} \alpha \mathrm{EW}$ in direct light converged to a value close to that from the FOS4 reflected light in 2014 and stalled afterwards (Fig. 4). EWs from both views remained at the same level during the last orbital cycle, suggesting that the coronagraph might have stopped vanishing. Alternatively, the coronagraph may have fully vanished, and this has happened $\sim 10 \mathrm{yr}$ earlier than previously predicted (Damineli et al. 2019). Spectra produced by CMFGEN modelling in the presence or not of an artificial coronagraph of varying sizes, shapes, and positions are in general agreement with observations (Fig. 10 and Table 2)

(v) The constancy of the $\mathrm{H} \delta$ and $\mathrm{H} \alpha$ EWs at the FOS4 reflected light reveals that the primary star in $\eta$ Car is more stable than has often been claimed, for example by Mehner et al. (2012). This indicates that whatever occurred during the GE, after $\sim$ two centuries the star has become stable again - see (Figs 3 and 4).

(vi) The variability of the coronagraph is not completely smooth as seen in the short-lived event that occurred at the end of the 1990's. 
This could be due to irregularities in the transparency of a moving occulter, or a temporary variation in the rate of vanishing (Fig. 9).

(vii) The variability curve of the $\mathrm{H} \alpha$ narrow absorption ('shell') line (Fig. 5) has a complex pattern, indicating that it is produced by a number of structures that may be moving across our LOS. All of these structures produce an ionization shadow in the LH at the position crossed by our LOS to the binary system. The non-periodic component of $\mathrm{H} \alpha$ narrow absorption ('shell') in Fig. 5 is produced by the coronagraph and it is weakening over the long term, in line with other evidence.

(viii) In addition to the coronagraph, there is a second kind of occulter that crosses our LOS to the binary system around periastron, when the ionizing secondary star is on the back side. This occulter is the primary's wind, which casts an ionization shadow to the LH at our LOS close to periastron. It produces the periodic component displayed in the variability curve of the $\mathrm{H} \alpha$ narrow absorption.

(ix) The fact that the disappearance of the coronagraph impacts the strength of absorption lines originating in the $\mathrm{LH}$ indicates that it is located inside the LH.

(x) Our best guess is that the coronagraph is a sheet-like a circumbinary disc seen at some inclination to our LOS - the NW border of which runs over the central stellar object and is extended to the SE as suggested by the Millour et al. (2020) and by Falcke et al. (1996) high spatial resolution polarimetric maps. The permanent $\mathrm{H} \alpha$ shell absorption reported by Boumis et al. (1998) could be produced by the same disc in the Homunculus equator. Partial occultation to the SE direction is suggested by the slow EW fading of Fe II $\lambda 4585$ reflected at FOS4. This evidence points to a $3 \mathrm{D}$ geometry of the occulter.

(xi) We could not identify the origin of the absorption feature at $\lambda 10792 \AA$. It is unlikely to be formed in the primary's stellar wind because there is no corresponding feature in any other spectral line of $\eta$ Car. It does not seem to be produced by the same DIB known in the ISM, since it has positively displaced absorption components to our LOS $\left(+45 \mathrm{~km} \mathrm{~s}^{-1}\right)$ and ISM DIBs are produced at much colder temperatures. Because its decreasing EW is tightly correlated with the decreasing extinction, we believe it is located inside the coronagraph, produced by an unidentified process/molecule.

The different molecular absorptions, with a range of velocities and equilibrium temperatures observed by ALMA indicate that there is a contribution of absorbers from a range of distances from the central star in our direction. Regarding the end of the changes in the coronagraph, if it will continue up to the year 2030, the brightness of the central star will surpass that of the situation previous to the GE. If this happens, taking into account the large quantity of mass shed during the GE, the primary star should have been rejuvenated and increased its luminosity. This would be in favour of the scenario of a binary merging in a triple stellar system. However, the question of how much such an internal mixing process could compensate for the lost mass can only be tackled by a specific model.

Significant progress to map the coronagraph could be made with high spatial resolution $(\sim$ mas) spectroscopy by sampling molecular absorption lines or resonant emission-lines.

\section{ACKNOWLEDGEMENTS}

AD thanks to Fundação de Amparo à Pesquisa do Estado de São Paulo (FAPESP) for support award numbers 2011/51680-6 and 2019/02029-2. FN acknowledges FAPESP (2017/18191-8). AFJM is grateful for financial aid to NSERC (Canada). MFC was supported by NASA under award number 80GSFC21M0002. We thank the referee A Mehner for many constructive criticisms.

\section{DATA AVAILABILITY}

The data underlying this article are available in the article and in its supplementary material. Full Tables A2-A5 are available at CDS via anonymous ftp to cdsarc.u-strasbg.fr (130.79.128.5) or via http: //cdsarc.u-strasbg.fr/viz-bin/qcat?J/MNRAS

\section{REFERENCES}

Allen D. A., Hillier D. J., 1993, Proc. Astron. Soc. Aust., 10, 338

Andriesse C. D., Donn B. D., Viotti R., 1978, MNRAS, 185, 771

Bordiu C., Rizzo J. R., 2019, MNRAS, 490, 1570

Boumis P., Meaburn J., Bryce M., Lopez J. A., 1998, MNRAS, 294, 61

Corcoran M. F. et al., 2017, ApJ, 838, 45

Damineli A., 1996, ApJ, 460, L49

Damineli A., Conti P. S., Lopes D. F., 1997, New Astron., 2, 107

Damineli A., Stahl O., Kaufer A., Wolf B., Quast G., Lopes D. F., 1998, A\&AS, 133, 299

Damineli A., Kaufer A., Wolf B., Stahl O., Lopes D. F., de Araújo F. X., 2000, ApJ, 528, L101

Damineli A. et al., 2008, MNRAS, 386, 2330

Damineli A. et al., 2019, MNRAS, 484, 1325

Damineli Neto A., Viotti R., Baratta G. B., de Araujo F. X., 1993, A\&A, 268, 183

Davidson K., Humphreys R. M., 1997, ARA\&A, 35, 1

Davidson K., Ebbets D., Weigelt G., Humphreys R. M., Hajian A. R., Walborn N. R., Rosa M., 1995, AJ, 109, 1784

Davidson K., Smith N., Gull T. R., Ishibashi K., Hillier D. J., 2001, AJ, 121, 1569

Davidson K. et al., 2005, AJ, 129, 900

Díaz-Luis J. J., García-Hernández D. A., Kameswara Rao N., Manchado A., Cataldo F., 2015, A\&A, 573, A97

Duncan R. A., White S. M., 2003, MNRAS, 338, 425

Espinoza Galeas D., Corcoran M. F., Hamaguchi K., Russell C., 2021, in American Astronomical Society Meeting Abstracts, 204.10

Falcke H., Davidson K., Hofmann K. H., Weigelt G., 1996, A\&A, 306, L17

Fernández-Lajús E. et al., 2010, New Astron., 15, 108

Grant D., Blundell K., Matthews J., 2020, MNRAS, 494, 17

Groh J. H., Damineli A., 2004, Inf. Bull. Var. Stars, 5492, 1

Groh J. H., Damineli A., Jablonski F., 2007, A\&A, 465, 993

Groh J. H. et al., 2010, A\&A, 517, A9

Groh J. H., Hillier D. J., Madura T. I., Weigelt G., 2012a, MNRAS, 423, 1623

Groh J. H., Madura T. I., Hillier D. J., Kruip C. J. H., Weigelt G., 2012b, ApJ, $759, \mathrm{~L} 2$

Gull T. R., Kober G. V., Nielsen K. E., 2006, ApJS, 163, 173

Gull T. R. et al., 2009, MNRAS, 396, 1308

Gull T. R. et al., 2016, MNRAS, 462, 3196

Hamano S. et al., 2015, ApJ, 800, 137

Hillier D. J., Allen D. A., 1992, A\&A, 262, 153

Hillier D. J., Miller D. L., 1998, ApJ, 496, 407

Hillier D. J., Davidson K., Ishibashi K., Gull T., 2001, ApJ, 553, 837

Hillier D. J. et al., 2006, ApJ, 642, 1098

Hirai R., Podsiadlowski P., Owocki S. P., Schneider F. R. N., Smith N., 2020, MNRAS, 503, 4276

Ishibashi K. et al., 2003, AJ, 125, 3222

Johansson S., Gull T. R., Hartman H., Letokhov V. S., 2005, A\&A, 435, 183

Madura T. I., Groh J. H., 2012, ApJ, 746, L18

Martin J. C., Koppelman M. D., 2004, AJ, 127, 2352

Martin J. C., Davidson K., Koppelman M. D., 2006, AJ, 132, 2717

Meaburn J., Walsh J. R., Wolstencroft R. D., 1993, A\&A, 268, 283

Mehner A., Davidson K., Ferland G. J., Humphreys R. M., 2010a, ApJ, 710, 729

Mehner A., Davidson K., Humphreys R. M., Martin J. C., Ishibashi K., Ferland G. J., Walborn N. R., 2010b, ApJ, 717, L22 
Mehner A., Davidson K., Humphreys R. M., Ishibashi K., Martin J. C., Ruiz M. T., Walter F. M., 2012, ApJ, 751, 73

Mehner A., Ishibashi K., Whitelock P., Nagayama T., Feast M., van Wyk F., de Wit W.-J., 2014, A\&A, 564, A14

Mehner A. et al., 2019, A\&A, 630, L6

Millour F. et al., 2020, preprint (arXiv:2006.15660)

Morris P. W. et al., 1999, Nature, 402, 502

Morris P. W., Gull T. R., Hillier D. J., Barlow M. J., Royer P., Nielsen K., Black J., Swinyard B., 2017, ApJ, 842, 79

Morris P. W. et al., 2020, ApJ, 892, L23

Navarete F. et al., 2020, Astron. Telegram, 13600, 1

Pickett C., Richardson N., Lane A., Strawn E., Gull T., 2021, in American Astronomical Society Meeting Abstracts, 136.04

Pittard J. M., Corcoran M. F., 2002, A\&A, 383, 636

Portegies Zwart S. F., van den Heuvel E. P. J., 2016, MNRAS, 456, 3401

Richardson N. D., Gies D. R., Henry T. J., Fernández-Lajús E., Okazaki A. T., 2010, AJ, 139, 1534

Richardson N. D., Gies D. R., Gull T. R., Moffat A. F. J., St-Jean L., 2015, AJ, 150, 109

Richardson N. D. et al., 2016, MNRAS, 461, 2540

Ruiz M. T., Melnick J., Ortiz P., 1984, ApJ, 285, L19

Sana H. et al., 2012, Science, 337, 444

Shull M., Darling J., Danforth C., 2021, Gaia-EDR3 Parallax Distances to the Great Carina Nebula and its Star Clusters (Trumpler 14, 15, 16). preprint (arXiv:2103.07922)

Smith N., Gehrz R. D., Hinz P. M., Hoffmann W. F., Hora J. L., Mamajek E. E., Meyer M. R., 2003a, AJ, 125, 1458

Smith N., Davidson K., Gull T. R., Ishibashi K., Hillier D. J., 2003, ApJ, 586, 432

Smith N. et al., 2018, MNRAS, 480, 1466

Stevens I. R., Blondin J. M., Pollock A. M. T., 1992, ApJ, 386, 265

Teodoro M. et al., 2012, ApJ, 746, 73

Teodoro M. et al., 2016, ApJ, 819, 131

Teodoro M., Gull T. R., Bautista M. A., Hillier D. J., Weigelt G., Corcoran M. F., 2020, MNRAS, 495, 2754

van Boekel R. et al., 2003, A\&A, 410, L37

Viotti R., Rossi L., Cassatella A., Altamore A., Baratta G. B., 1989, ApJS, 71,983

Weigelt G., Ebersberger J., 1986, A\&A, 163, L5

Weigelt G., Kraus S., 2012, High-Resolution Studies of Eta Carinae's Ejecta and Stellar Wind, Vol. 384, Astrophysics and Space Science Library Springer, p. 129

Weigelt G. et al., 1995, in Niemela V., Morrell N., Feinstein A., eds, Revista Mexicana de Astronomia y Astrofisica Conference Series Vol.
2, Revista Mexicana de Astronomia y Astrofisica Conference Series, La Plata Argentina. p. 11

Weigelt G. et al., 2007, A\&A, 464, 87

Zanella R., Wolf B., Stahl O., 1984, A\&A, 137, 79

\section{SUPPORTING INFORMATION}

Supplementary data are available at $M N R A S$ online.

Table S3. EWs in direct light for $\mathrm{H} \alpha$

Table S4. EWs in direct light for Fe II $\lambda 4585$.

Table S5. EWs in direct star for $\mathrm{H} \delta$.

Please note: Oxford University Press is not responsible for the content or functionality of any supporting materials supplied by the authors. Any queries (other than missing material) should be directed to the corresponding author for the article.

\section{APPENDIX A: EXTENDED DATA TABLES}

Table A1. DIB1 and DIB2 EWs in normal and emission-line stars.

\begin{tabular}{lccccc}
\hline Object & $\begin{array}{c}\text { EW } \\
\lambda 10780 \\
(\mathrm{~m} \AA)\end{array}$ & $\begin{array}{c}\sigma_{\mathrm{EW}} \\
(\mathrm{m} \AA)\end{array}$ & $\begin{array}{c}\text { EW } \\
\lambda 10792 \\
(\mathrm{~m} \AA)\end{array}$ & $\begin{array}{c}\sigma_{\mathrm{EW}} \\
(\mathrm{m} \AA)\end{array}$ & $\begin{array}{c}\text { Spectral } \\
\text { type }\end{array}$ \\
\hline$\beta$ Monocerotis & 72 & 10 & 66 & 10 & B4Ve \\
$\delta$ Scorpii & 180 & 20 & 136 & 18 & B0.3IVe \\
$\beta$ Lyrae & 82 & 12 & 56 & 9 & B8.5Ib-II \\
HD 169454 & 169 & 18 & 86 & 11 & A1V \\
AG Carinae & 130 & 15 & 66 & 9 & WN11h- \\
& & & & & LBV \\
$\chi$ Ophiuci & 94 & 13 & 46 & 8 & B2Vne \\
HD 168625 & 242 & 30 & 110 & 15 & B6Iap-LBV \\
HR Carinae & 196 & 27 & 89 & 16 & LBV \\
HD 168607 & 221 & 18 & 74 & 10 & B9Iaep- \\
& & & & & LBV \\
MWC 314 & 267 & 30 & 87 & 12 & B3Ibe-LBV \\
P Cygni & 91 & 16 & 29 & 7 & LBV \\
HD 152236 & 61 & 16 & 19 & 7 & B1Ia-0ek \\
HD 316285 & 387 & 100 & 90 & 20 & B0Ieq-LBV \\
WD1-W243 & 690 & 120 & 134 & 30 & LBV \\
\hline
\end{tabular}


Table A2. EW of the $\lambda 10792 \AA$ absorption in eta Carinae observed at OPD/Coudé.

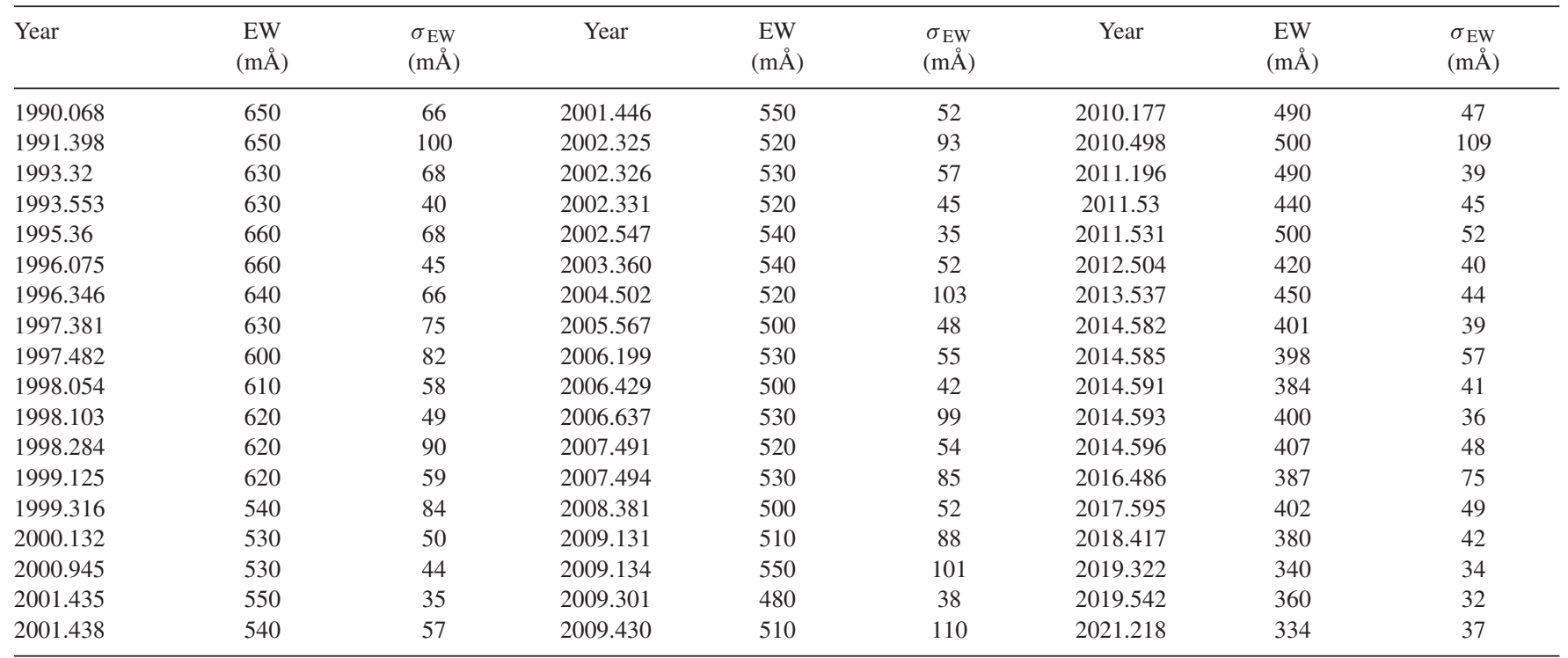

Table A3. EWs in direct light for $\mathrm{H} \alpha$ (first 10 rows). The full table is available online as supplementary material and in the CDS.

\begin{tabular}{lccc}
\hline HJD & $\begin{array}{c}\text { EW H } \alpha \\
(\AA)\end{array}$ & $\begin{array}{c}\sigma_{\text {EW }} \\
(\AA)\end{array}$ & Site \\
\hline 49063.609 & -1009 & 48 & ESO/FEROS \\
49065.609 & -1013 & 51 & ESO/FEROS \\
49066.621 & -996 & 49 & ESO/FEROS \\
49067.605 & -1005 & 50 & ESO/FEROS \\
49068.609 & -1001 & 52 & ESO/FEROS \\
49069.609 & -1009 & 50 & ESO/FEROS \\
49071.613 & -1006 & 50 & ESO/FEROS \\
49072.613 & -962 & 52 & ESO/FEROS \\
49073.648 & -1004 & 49 & ESO/FEROS \\
49074.625 & -998 & 49 & ESO/FEROS \\
\hline
\end{tabular}

Table A4. EW in direct light for Fe II $\lambda 4585$ (first 10 rows). The full table is available online as supplementary material and in the CDS.

\begin{tabular}{lccc}
\hline HJD & $\begin{array}{c}\text { EW Fe II } \lambda 4585 \\
(\AA)\end{array}$ & $\begin{array}{c}\sigma_{\text {EW }} \\
(\AA)\end{array}$ & Site \\
\hline 48844.4880 & -16.1 & 1.8 & ESO/FEROS \\
49063.6090 & -15.2 & 1.7 & ESO/FEROS \\
49067.6050 & -16.0 & 1.7 & ESO/FEROS \\
49069.6090 & -16.0 & 2.8 & ESO/FEROS \\
49071.6130 & -16.1 & 1.5 & ESO/FEROS \\
49072.6130 & -15.0 & 3.2 & ESO/FEROS \\
49073.6480 & -15.3 & 1.3 & ESO/FEROS \\
49075.5740 & -15.2 & 2.3 & ESO/FEROS \\
49076.6250 & -15.0 & 2.7 & ESO/FEROS \\
49077.5900 & -15.4 & 2.3 & ESO/FEROS \\
\hline
\end{tabular}

Table A5. EWs in direct star for $\mathrm{H} \delta$ (first 10 rows). The full table is available online as supplementary material and in the CDS.

\begin{tabular}{lccc}
\hline HJD & $\begin{array}{c}\text { EW H } \delta \\
(\AA)\end{array}$ & $\begin{array}{c}\sigma_{\text {EW }} \\
(\AA)\end{array}$ & Site \\
\hline 58502.451 & -27.4 & 1.0 & LCO/NRES \\
59039.234 & -25.4 & 2.0 & LCO/NRES \\
59051.232 & -26.4 & 0.6 & LCO/NRES \\
59152.602 & -24.1 & 1.4 & LCO/NRES \\
59155.600 & -25.0 & 1.2 & LCO/NRES \\
59174.562 & -24.6 & 0.8 & LCO/NRES \\
59206.513 & -23.8 & 1.1 & LCO/NRES \\
59210.463 & -24.5 & 0.9 & LCO/NRES \\
49063.609 & -28.8 & 13.5 & ESO/FEROS \\
49065.609 & -32.5 & 12.2 & ESO/FEROS \\
\hline
\end{tabular}


Table A6. EWs of $\mathrm{H} \alpha \mathrm{H} \delta$ and Fe II $\lambda 4585$ from UVES observations extracted at FOS4 position.

\begin{tabular}{|c|c|c|c|c|c|c|c|c|c|c|c|}
\hline JD-2400000 & Year & $\begin{array}{c}\mathrm{EW} \mathrm{H} \delta \\
(\AA)\end{array}$ & $\begin{array}{c}\sigma_{\mathrm{EW}} \\
(\AA)\end{array}$ & JD-2400000 & Year & $\begin{array}{c}\text { EW Fe II } \lambda 4585 \\
(\AA)\end{array}$ & $\begin{array}{l}\sigma_{\mathrm{EW}} \\
(\AA)\end{array}$ & JD-2400000 & Year & $\begin{array}{c}\mathrm{EW} \mathrm{H} \alpha \\
(\AA)\end{array}$ & $\begin{array}{l}\sigma_{\mathrm{EW}} \\
(\AA)\end{array}$ \\
\hline 51533.832 & 1999.97 & -22.00 & 0.61 & 51533.832 & 1999.97 & -7.00 & 0.49 & 52616.833 & 2002.94 & -521 & 8 \\
\hline 52639.840 & 2003.00 & -21.00 & 0.57 & 52662.862 & 2003.06 & -6.92 & 1.38 & 52642.832 & 2003.01 & -555 & 9 \\
\hline 52662.862 & 2003.06 & -19.60 & 0.61 & 52674.862 & 2003.09 & -6.90 & 0.21 & 52658.814 & 2003.05 & -530 & 9 \\
\hline 52662.862 & 2003.06 & -19.53 & 0.61 & 52990.816 & 2003.96 & -5.90 & 0.36 & 52662.848 & 2003.06 & -532 & 9 \\
\hline 52968.807 & 2003.90 & -20.04 & 0.73 & 53029.774 & 2004.07 & -5.55 & 0.38 & 52674.848 & 2003.09 & -530 & 9 \\
\hline 53029.774 & 2004.07 & -20.33 & 0.67 & 53075.562 & 2004.19 & -6.91 & 0.46 & 52990.803 & 2003.96 & -564 & 6 \\
\hline 53055.653 & 2004.14 & -21.32 & 0.67 & 53389.738 & 2005.05 & -6.50 & 0.58 & 53029.763 & 2004.07 & -540 & 7 \\
\hline 53075.562 & 2004.19 & -20.56 & 0.93 & 53431.818 & 2005.17 & -6.18 & 0.48 & 53075.703 & 2004.19 & -564 & 9 \\
\hline 53389.738 & 2005.05 & -19.08 & 0.82 & 53866.524 & 2006.36 & -6.68 & 0.51 & 53349.850 & 2004.94 & -535 & 11 \\
\hline 53431.818 & 2005.17 & -19.50 & 0.66 & 53912.550 & 2006.48 & -6.84 & 0.55 & 53389.709 & 2005.05 & -540 & 14 \\
\hline 53866.524 & 2006.36 & -19.88 & 0.72 & 54535.755 & 2008.19 & -6.67 & 0.41 & 53389.725 & 2005.05 & -541 & 8 \\
\hline 54583.543 & 2008.32 & -20.88 & 0.64 & 54657.552 & 2008.52 & -5.35 & 0.47 & 54583.529 & 2008.32 & -543 & 14 \\
\hline 54599.492 & 2008.36 & -20.46 & 0.63 & 54999.565 & 2009.46 & -4.34 & 0.39 & 54599.475 & 2008.36 & -552 & 14 \\
\hline 54629.491 & 2008.45 & -20.08 & 0.67 & 55013.483 & 2009.50 & -4.86 & 0.34 & 54616.536 & 2008.41 & -547 & 13 \\
\hline 54657.552 & 2008.52 & -20.20 & 0.66 & 56620.846 & 2013.90 & -4.00 & 0.71 & 54629.504 & 2008.45 & -529 & 13 \\
\hline 54999.565 & 2009.46 & -20.05 & 0.67 & 56650.835 & 2013.98 & -4.31 & 0.71 & 56999.838 & 2014.94 & -485 & 36 \\
\hline 55013.483 & 2009.50 & -20.00 & 0.44 & 56684.722 & 2014.07 & -4.05 & 0.61 & 56999.841 & 2014.94 & -487 & 12 \\
\hline 56620.846 & 2013.90 & -21.00 & 1.26 & 56712.686 & 2014.15 & -4.28 & 0.74 & 57012.805 & 2014.97 & -505 & 13 \\
\hline 57043.865 & 2015.06 & -19.08 & 1.39 & 57043.865 & 2015.06 & -3.68 & 0.82 & 57027.837 & 2015.01 & -497 & 27 \\
\hline 57047.796 & 2015.07 & -19.63 & 0.99 & 57047.796 & 2015.07 & -3.87 & 0.59 & 57047.781 & 2015.07 & -531 & 13 \\
\hline
\end{tabular}

This paper has been typeset from a $\mathrm{T}_{\mathrm{E}} \mathrm{X} / \mathrm{LT} \mathrm{E} \mathrm{X}$ file prepared by the author. 